\title{
CONVOLUTION, DIFFERENTIAL EQUATIONS, AND ENTIRE FUNCTIONS OF EXPONENTIAL TYPE $\left({ }^{1}\right)$ \\ BY
}

\author{
DALE H. MUGLER
}

ABSTRACT. The Whittaker-Shannon interpolation formula, or "cardinal series", is a special case of the more general linear integro-differential equation with constant complex coefficients $\Sigma_{n=0}^{\infty} a_{n} f^{(n)}(z)=\int f(z-t) d \mu(t)$ where the integral is taken over the whole real line with respect to the measure $\mu$.

In this study, I show that many of these equations provide representations for particular classes of entire functions of exponential type. That is, every function in the class satisfies the equation and conversely every solution of the equation is a member of the class of functions.

When the measure in the convolution integral above is chosen to be discrete, a particular form of the above type of equation is an equation of periodicity $f(z)=f(z+\tau)$. Following an extensive treatment of the general equation written above, the study concludes by offering a generalization in terms of these convolution equations of a classical theorem in complex analysis concerning periodic entire functions.

1. Introduction. An entire function $f(z)$ which satisfies the condition $|f(z)| \leqslant A e^{k|z|}$ for nonnegative constants $A$ and $k$ is called an entire function of exponential type. Any such function which is also square-integrable on the real line is known to satisfy the Whittaker-Shannon interpolation formula [22], the so-called "cardinal series", from which the function can be reconstructed by knowing only the values it takes at certain points along the real axis. A special case of this formula is the equation

$$
f(z)=\sum_{n=-\infty}^{+\infty} \frac{\sin \pi(z-n)}{\pi(z-n)} f(n)
$$

for all complex $z$.

The Whittaker-Shannon interpolation formula is a special case of the more general linear integro-differential equation with constant complex coefficients

Received by the editors September 18, 1974.

AMS (MOS) subject classifications (1970). Primary 30A62, 30A64, 30A80, 34A20, 34A35, 34J 10, 44A35, 45J05.

Key words and phrases. Entire function, exponential type, interpolation, integrodifferential equation, differential-difference equation, convolution, Laplace transform, distribution.

(1) This work is based on material contained in the author's doctoral dissertation, which was completed at Northwestern University under the direction of Professor R. P. Boas, Jr. 


$$
\sum_{n=0}^{\infty} a_{n} f^{(n)}(z)=\int f(z-t) d \mu(t)
$$

where the integral is taken over the real line with respect to the nontrivial measure $\mu$. When the measure is chosen to be discrete, other special cases of this equation are equations of periodicity $f(z)=f(z+\tau)$ or more general linear differential-difference equations of the form $\Sigma_{n=0}^{N} a_{n} f^{(n)}(z)=\Sigma_{k=0}^{\infty} f\left(z+\tau_{k}\right)$, where $\tau_{k}$ is real for $k \geqslant 0$.

This study shows that many of these equations characterize certain classes of entire functions of exponential type, i.e., every function in the class satisfies the particular equation and, conversely, every solution of that equation is a member of the class of functions.

We first investigate these convolution equations in the case that the measure itself has a finite Laplace transform on some interval of the real line. When the interval is symmetric about the origin, we show that the convolution integral represents a linear differential operator which is related to the measure by its bilateral Laplace transform. Conversely, we show that the only possible solutions of equation (a), where the function $\Sigma_{n=0}^{\infty} a_{n} z^{n}$ is transcendental and analytic in $|z| \leqslant a$, are entire functions of exponential type $a$, and thus we obtain a representation for these functions. Whether $\Sigma_{n=0}^{\infty} a_{n} z^{n}$ is transcendental or not, we exhibit a method by which solutions of (a) can be constructed. Finally, where the conditions are as described in Proposition 2.6, we show that all solutions which are entire of exponential type have a representation as sums of exponential functions.

In the third section, we discuss the behavior of the solutions of

$$
\sum_{n=0}^{N} a_{n} f^{(n)}(x)=\int_{-\infty}^{+\infty} f(x-t) d \mu(t)
$$

where $N$ is finite and the Laplace transform of the measure may not exist in any interval. Interpolation methods between derivatives are used to find that, in general, the solution is necessarily an entire function with exponential type depending on the magnitude of the coefficients $a_{n}$, the order $N$ of the equation, and the $L^{1}$ norm with respect to the measure $|\mu|$ of a certain function depending only on the solution.

The next section begins with a theorem showing that any bounded linear operator acting on the space of entire functions of exponential type $a$ which are square-integrable on the real line (a space denoted $W_{a}$ ) has a representation as a convolution. This space of functions, the "band-limited" functions, is important in information theory where the cardinal series plays an important role.

We proceed to develop conditions on the measure such that equations of the form (b) characterize the class $W_{a}$, and exhibit several examples of such equations. This section continues by considering the equations characterizing entire 
functions of exponential type which have polynomial growth on the real line. The theory of distributions plays an important part, and we are again able to classify the measures and hence the particular equations involved. The section closes by considering an equivalent condition characterizing equations of the form (b) in terms of the moments of the measure.

This study concludes by offering a generalization for this class of entire functions of one of the classical theorems in complex analysis.

Note. The large majority of integrals in this paper are considered over the whole real line. Thus the integrals are to be considered in this manner unless otherwise indicated.

2. Convolution and differential equations for the class of entire functions of exponential type. We first characterize the form of the convolution integral.

THEOREM 2.1. Let $f(z)$ be entire of exponential type a. Suppose that $\mu$ is a measure such that $e^{\sigma|x|} \in L^{1}(d|\mu|)$ for some $\sigma>a$. Then

$$
\int f(x-t) d \mu(t)=\sum_{n=0}^{\infty} \lambda_{n} f^{(n)}(x)
$$

where $\lambda(z)=\sum_{n=0}^{\infty} \lambda_{n} z^{n}$ is analytic in $|z| \leqslant a$, and $\lambda$ and $\mu$ are related by the formula $\lambda(z)=\int e^{-z t} d \mu(t)$, i.e., by the bilateral Laplace transform. Conversely, if $\lambda(z)$ is related to a measure in this fashion, then (1) holds.

Proof. We first define $T_{\mu}$ by $T_{\mu}[f]=\int f(z-t) d \mu(t)$ where $f$ is entire of exponential type $a$. By the hypothesis, we know that the integral is well defined for all $z$, since

$$
\begin{aligned}
\left|\int f(z-t) d \mu(t)\right| & \leqslant \int A e^{(a+\epsilon)|z-t|} d|\mu|(t) \\
& \leqslant A e^{(a+\epsilon)|z|} \int e^{(a+\epsilon)|t|} d|\mu|(t)<\infty
\end{aligned}
$$

for any $\dot{\epsilon}>0$ such that $a+\epsilon<\sigma$.

To show that $T_{\mu}[f]$ is an entire function, let $C_{r}$ be the circle $|z|=r$ for $r \geqslant 0$, and consider

$$
\int_{C_{r}} T_{\mu}[f](z) d z=\int_{C_{r}} \int f(z-t) d \mu(t) d z=\iint_{C_{r}} f(z-t) d z d \mu(t) .
$$

But $g_{t}(z)=f(z-t)$ is entire since $f(z)$ is, for any fixed $t$. Thus Cauchy's Theorem implies $\int_{C_{r}} g_{t}(z) d z=0$ for any $r$ and any $t$. But then

$$
\int_{C_{r}} T_{\mu}[f](z) d z=\int 0 \cdot d \mu(t)=0
$$

for any circle $r$. Thus Morera's Theorem applies to say that $T_{\mu}[f]$ is entire. 
Moreover, the growth relation demonstrated above combined with the hypothesis that $\int e^{(a+\epsilon)|t|} d|\mu|(t)<\infty$ shows that $T_{\mu}[f]$ is entire of exponential type.

We wish to apply a theorem of [2], and we thus need to show that $T_{\mu}$ is (i) distributive, (ii) commutes with differentiation, and (iii) satisfies the weak continuity condition defined below.

$$
\begin{aligned}
T_{\mu}[a f(z)+b g(z)] & =\int\{a f+b g\}(z-t) d \mu(t) \\
& =a \int f(z-t) d \mu(t)+b \int g(z-t) d \mu(t) \\
& =a T_{\mu}[f(z)]+b T_{\mu}[g(z)]
\end{aligned}
$$

so that $T_{\mu}$ is distributive.

$$
\frac{d}{d z}\left[\int f(z-t) d \mu(t)\right]=\int f^{\prime}(z-t) d \mu(t)
$$

follows from Lemma 1 of $\S 3$ below, so that $\left[T_{\mu}[f]\right]^{\prime}=T_{\mu}\left[f^{\prime}\right]$.

(iii) The condition we need to show is the following: Let $s_{n}(z)=\sum_{k=0}^{n} a_{k} z^{k}$ where $f(z)=\Sigma_{k=0}^{\infty} a_{k} z^{k}$ is an entire function of exponential type $a$. Then $\lim _{n \rightarrow \infty} T_{\mu}\left[s_{n}(z)\right]=T_{\mu}[f(z)]$ for every $z$.

To show this, fix $z$ complex. Since $f(z)$ is entire of exponential type $a$, it is clear that for every $n$ there exists some number $\epsilon(0<\epsilon<\sigma-a)$ such that $\left|s_{n}(z-t)\right| \leqslant \exp \{(a+\epsilon)[|z|+|t|]\}$ for all real $t$, and $\epsilon$ does not depend on $n$. This latter function is by hypothesis in $L^{1}(d|\mu|)$. Thus

$$
\lim _{n \rightarrow \infty} \int \sum_{k=0}^{n} a_{k}(z-t)^{k} d \mu(t)=\int \sum_{k=0}^{\infty} a_{k}(z-t)^{k} d \mu(t)=\int f(z-t) d \mu(t)
$$

follows from the dominated convergence theorem. This shows (iii).

Theorem 1 of [2] now applies. Hence $T_{\mu}$ has the form designated in the theorem, i.e., $T_{\mu}[f(z)]=\Sigma_{n=0}^{\infty} \lambda_{n} f^{(n)}(z)$ for some $\lambda(z)=\sum_{n=0}^{\infty} \lambda_{n} z^{n}$ analytic in $|z| \leqslant a$.

To show that $\lambda(z)=\int e^{-z t} d \mu(t)$, let $z_{0}$ be any fixed number such that $\left|z_{0}\right|$ $\leqslant a$. Form the entire function of exponential type $a$ given by $\exp \left(z_{0} z\right)$ for complex $z$. Since (1) holds for this function:

$$
\begin{aligned}
& \int \exp \left(z_{0}(x-t)\right) d \mu(t)=\sum_{n=0}^{\infty} \lambda_{n} \frac{d^{n}}{d x^{n}} \exp \left(z_{0} x\right) \\
& \Rightarrow e^{z 0^{x}} \int e^{-z 0^{t}} d \mu(t)=e^{z 0^{x}} \sum_{n=0}^{\infty} \lambda_{n} z_{0}^{n} \Rightarrow \int e^{-z 0^{t}} d \mu(t)=\sum_{n=0}^{\infty} \lambda_{n} z_{0}^{n} .
\end{aligned}
$$

This holds for every $z_{0}$ such that $\left|z_{0}\right| \leqslant a$, so $\lambda(z)$ has the required form.

Conversely, suppose that $\lambda(z)=\sum_{n=0}^{\infty} \lambda_{n} z^{n}$ is analytic in $|z| \leqslant a$ and that $\lambda(z)$ is the Laplace transform of a measure with the hypothesized properties.

Then $\lambda(z)$ is actually analytic in $|z| \leqslant \sigma$. 
Let $f(z)$ be entire of exponential type $a$, and let $F(z)$ be its Borel transform. It is well known for every such function that

$$
f(z)=\frac{1}{2 \pi i} \int_{|\omega|=a+\epsilon} F(\omega) e^{z \omega} d \omega
$$

where $\epsilon>0$ is chosen so that $a+\epsilon<\sigma$. Thus

$$
\begin{aligned}
\int f(z-t) d \mu(t) & =\frac{1}{2 \pi i} \iint_{|\omega|=a+\epsilon} F(\omega) e^{z \omega-t \omega} d \omega d \mu(t) \\
& =\frac{1}{2 \pi i} \int_{|\omega|=a+\epsilon} \int F(\omega) e^{z \omega-t \omega} d \mu(t) d \omega \\
& =\frac{1}{2 \pi i} \int_{|\omega|=a+\epsilon} F(\omega) e^{z \omega}\left\{\int e^{-t \omega} d \mu(t)\right\} d \omega \\
& =\frac{1}{2 \pi i} \int_{|\omega|=a+\epsilon} F(\omega) e^{z \omega} \sum_{n=0}^{\infty} \lambda_{n} \omega^{n} d \omega .
\end{aligned}
$$

But $\lambda(\omega)=\sum_{n=0}^{\infty} \lambda_{n} \omega^{n}$ is analytic in a disk containing this contour, so the integration may be carried out term by term. That is,

$$
\int f(z-t) d \mu(t)=\sum_{n=0}^{\infty} \lambda_{n} \cdot \frac{1}{2 \pi i} \int_{|\omega|=a+\epsilon} F(\omega) \omega^{n} e^{z \omega} d \omega=\sum_{n=0}^{\infty} \lambda_{n} f^{(n)}(z)
$$

as stated.

We will proceed now to show that the solutions of equations of the form (1) are infinitely differentiable and, in general, are entire functions of exponential type. The cases where $\lambda(z)$ in Theorem 2.1 is a polynomial are considered in Propositions 3.4 and 3.5. First, however, we consider the case where $\lambda(z)$ is transcendental.

Proposition 2.2. Suppose that (1) holds for some $f(x) \in C^{\infty}(R)$, where $\lambda(z)$ is a transcendental function which is analytic in $|z| \leqslant a$. Then $f(z)$ is an entire function of exponential type a.

REMARK. Sikkema [20] states that a "necessary and sufficient condition for the differential operator $F(D)=\Sigma_{n=0}^{\infty} a_{n} D^{n}$ to be applicable to all entire functions of type $\tau$ and order 1 is that the numbers $a_{n}$ are such that the generating power series $\sum_{n=0}^{\infty} a_{n} z^{n}$ defines a function which is analytic for $|z| \leqslant \tau$ ". Our assumptions on $\lambda(z)$ therefore appear to be quite necessary.

ProOF. Let $x$ be fixed. The convergence of $\Sigma_{n=0}^{\infty} a_{n} f^{(n)}(x)$ implies that $\left(\right.$ for $\lambda(z)=\Sigma_{n=0}^{\infty} a_{n} z^{n}$ in (1))

$$
\left|a_{n} f^{(n)}(x)\right| \rightarrow 0 \quad \text { as } n \rightarrow \infty .
$$

Thus $\left|a_{n} f^{(n)}(x)\right|<1$ for all $n \geqslant N_{1}$. 
The function $\lambda(z)=\Sigma_{n=0}^{\infty} a_{n} z^{n}$ is analytic in $|z| \leqslant a$, so that $\overline{\lim }_{n \rightarrow \infty}\left|a_{n}\right|^{1 / n}$ $\leqslant 1 / a$. Thus, for arbitrary $\epsilon>0$,

$$
\left|a_{n}\right|^{1 / n}>1 / a-\epsilon \text { for all } n \geqslant N_{2} \geqslant N_{1} .
$$

Thus

$$
\begin{aligned}
& (1 / a-\epsilon)^{n}\left|f^{(n)}(x)\right|<1 \text { for all } n \geqslant N_{2} \\
& \quad \Rightarrow\left|f^{(n)}(x)\right|<(1 / a-\epsilon)^{-n} \text { for all } n \geqslant N_{2} \\
& \quad \Rightarrow\left|f^{(n)}(x)\right|^{1 / n}<(1 / a-\epsilon)^{-1} \text { for all } n \geqslant N_{2} .
\end{aligned}
$$

Thus $\lim \sup _{n \rightarrow \infty}\left|f^{(n)}(x)\right|^{1 / n}<(1 / a-\epsilon)^{-1}$, and as $\epsilon>0$ was arbitrary,

$$
\limsup _{n \rightarrow \infty}\left|f^{(n)}(x)\right|^{1 / n} \leqslant(1 / a)^{-1}=a .
$$

Define a function $g(z)=\Sigma_{n=0}^{\infty} f^{(n)}(0) z^{n} / n$ !. This is an entire function, since

$$
\underset{n \rightarrow \infty}{\limsup }\left|\frac{f^{(n)}(0)}{n !}\right|^{1 / n} \leqslant \limsup _{n \rightarrow \infty}\left|\frac{(1 / a-\epsilon)^{-n}}{n !}\right|^{1 / n}=0 .
$$

Further, (2) shows that $g(z)$ is of exponential type $a$.

We claim that $g(x)=\Sigma_{n=0}^{\infty} f^{(n)}(0) x^{n} / n$ ! is the Taylor series expansion for the original function $f(x)$. This will be true if the remainder term

$$
R_{n}(x, 0)=\int_{0}^{x} \frac{(x-t)^{n}}{n !} f^{(n+1)}(t) d t
$$

goes to zero as $n$ goes to $\infty$.

Now

$$
\begin{aligned}
\left|R_{n}(x, 0)\right| & \leqslant \int_{0}^{x} \frac{|x-t|^{n}}{n !}\left|f^{(n+1)}(t)\right| d t \\
& \leqslant \max _{0 \leqslant|t| \leqslant|x|}\left|f^{(n+1)}(t)\right| \frac{|x|^{n+1}}{(n+1) !} .
\end{aligned}
$$

For each $t, 0 \leqslant|t| \leqslant|x|$, the above analysis shows that there is an $N_{t}$ such that $\left|f^{(n+1)}(t)\right|<(1 / a-\epsilon)^{-(n+1)}$ for $n \geqslant N_{t}$ and $\epsilon>0$ such that $\epsilon<1 / a$. In fact, picking $N_{1}$ according to the above analysis shows that there is some neighborhood about each $t$ such that a particular $N$ will do. But $[0, x]$ is compact, so that there is some $N^{\prime}$ independent of $t$ such that $\left|f^{(n+1)}(t)\right|<(1 / a-\epsilon)^{-(n+1)}$ for $n \geqslant N^{\prime}$ and $0 \leqslant|t| \leqslant|x|$. Thus the latter expression above goes to zero as $n$ tends to $\infty$, so $R_{n}(x, 0)$ goes to zero as $n$ tends to $\infty$.

Thus extending $f(x)$ to the plane by $f(z)=g(z)$ completes the proof.

EXAMPLES.

EXAmple 1. Consider the equation

$$
\sum_{n=0}^{\infty} f^{(2 n)}(x)=\int f(x-t)^{1 / 2} e^{-|t|} d t
$$


If $d \mu(t)=1 / 2 e^{-|t|} d t$ in Theorem 2.1 , then by Laplace transforms

$$
\int e^{-z t} d \mu(t)=\frac{1}{1-z^{2}}=\sum_{0}^{\infty} z^{2 n} \text { for }|z|<1,
$$

so Theorem 2.1 shows that (3) is satisfied by every entire function of exponential type less than 1 .

Conversely, Proposition 2.2 shows that for any $f \in C^{\infty}(R)$ such that (3) holds, $f$ is entire of exponential type at most 1 .

Thus combining the theorem and the proposition shows that equation (3) characterizes entire functions of exponential type less than 1.

EXAMPLE 2. In a similar manner, we see that the more general equation

$$
\sum_{n=0}^{\infty}\left(\frac{1}{a}\right)^{2 n} f^{(2 n)}(x)=\int f(x-t) \frac{a}{2} e^{-a|t|} d t
$$

characterizes entire functions of exponential type less than $a$.

We proceed now to develop a method by which to construct solutions of (1). We first consider the solutions of equations of the form

$$
\sum_{n=0}^{K} b_{n} f^{(n)}(x)=\int f(x-t) d \mu(\dot{t})
$$

where $\int e^{\sigma|t|} d|\mu|(t)<\infty$ for some $\sigma>0$, and $K$ is finite.

Proposition 2.3. If $z=u_{0}$ is a zero of multiplicity $N$ of the function

$$
g(z)=\sum_{n=0}^{K} b_{n} z^{n}-\int e^{-z t} d \mu(t),
$$

then $f(x)=P(x) e^{u_{0} x}$, where $P(x)$ is any polynomial of degree at most $N-1$, is $a$ solution of (5).

Proof. We note that $z=u_{0}$ satisfies

$$
\int(-t)^{j} e^{-z t} d \mu(t)=\left\{\begin{array}{l}
\sum_{n=j}^{K} j !\left(\begin{array}{l}
n \\
j
\end{array}\right) b_{n} z^{n-j} \quad \text { if } 0 \leqslant j \leqslant K, \\
0 \quad \text { if } K<j \leqslant N .
\end{array}\right.
$$

Let $P(x)=\Sigma_{m=0}^{N-1} a_{m} x^{m}$, so that

$$
\begin{aligned}
\int f(x-t) d \mu(t) & =\int \sum_{m=0}^{N-1} a_{m}(x-t)^{m} e^{u_{0}(x-t)} d \mu(t) \\
& =\sum_{m=0}^{N-1} a_{m} e^{u_{0}^{x}} \int(x-t)^{m} e^{-u_{0} t} d \mu(t) \\
& =\sum_{m=0}^{N-1} a_{m} e^{u_{0}^{x}} \sum_{j=0}^{m}(-1)^{j}\left(\begin{array}{c}
m \\
j
\end{array}\right) x^{m-j} \int t^{j} e^{-u_{0} t} d \mu(t)
\end{aligned}
$$


where we used the binomial theorem to expand $(x-t)^{m}$ :

$$
(x-t)^{m}=\sum_{j=0}^{m}(-1)^{j}\left(\begin{array}{c}
m \\
j
\end{array}\right) x^{m-j} t^{j}
$$

Using the hypothesis,

$$
\begin{aligned}
\int f(x-t) d \mu(t) & =e^{u_{0} x} \sum_{m=0}^{N-1} \sum_{j=0}^{m} a_{m}\left(\begin{array}{c}
m \\
j
\end{array}\right) x^{m-j}\left[\sum_{n=j}^{K} j !\left(\begin{array}{l}
n \\
j
\end{array}\right) b_{n} u_{0}^{n-j}\right] \\
& =e^{u 0^{x}} \sum_{m=0}^{N-1} \sum_{j=0}^{m} \sum_{n=j}^{K} a_{m}\left(\begin{array}{c}
m \\
j
\end{array}\right) j !\left(\begin{array}{c}
n \\
j
\end{array}\right) b_{n} x^{m-j} u_{0}^{n-j} \\
& =e^{u 0^{x}} \sum_{n=0}^{K} \sum_{j=0}^{n} \sum_{m=j}^{N-1} a_{m}\left(\begin{array}{c}
m \\
j
\end{array}\right) j !\left(\begin{array}{c}
n \\
j
\end{array}\right) b_{n} x^{m-j} u_{0}^{n-j}
\end{aligned}
$$

Claim.

(6) $\frac{d^{n}}{d x^{n}}\left\{\sum_{m=0}^{N-1} a_{m} x^{m} e^{u 0^{x}}\right\}=e^{u_{0} x} \sum_{j=0}^{n} \sum_{m=j}^{N-1} a_{m}\left(\begin{array}{c}m \\ j\end{array}\right) j !\left(\begin{array}{c}n \\ j\end{array}\right) x^{m-j} u_{0}^{n-j}$.

Proof (Induction on $n$ ). $n=0$. Then

$$
\sum_{m=0}^{N-1} a_{m} x^{m} e^{u_{0} x}=e^{u_{0} x} \sum_{m=0}^{N-1} a_{m}\left(\begin{array}{l}
m \\
0
\end{array}\right) 0 !\left(\begin{array}{l}
0 \\
0
\end{array}\right) x^{m-0} u_{0}^{n-0}=e^{u_{0} x} \sum_{m=0}^{N-1} a_{m} x^{m}
$$

so that (6) holds in this case.

Now suppose that (6) holds for $n=p$. Thus

$$
\begin{aligned}
\frac{d^{p+1}}{d x^{p+1}}\left\{\sum_{m=0}^{N-1} a_{m} x^{m} e^{u} 0^{x}\right\} & \\
= & \frac{d}{d x}\left\{e^{u} 0^{x} \sum_{j=0}^{p} \sum_{m=j}^{N-1} a_{m}\left(\begin{array}{c}
m \\
j
\end{array}\right) j !\left(\begin{array}{l}
p \\
j
\end{array}\right) x^{m-j} u_{0}^{p-j}\right\} \\
= & e^{u} 0^{x}\left[\begin{array}{l}
u_{0} \\
\sum_{j=0}^{p} \sum_{m=j}^{N-1} a_{m}\left(\begin{array}{c}
m \\
j
\end{array}\right) j !\left(\begin{array}{l}
p \\
j
\end{array}\right) x^{m-j} u_{0}^{p-j}
\end{array}\right. \\
& \left.+\sum_{j=0}^{p} \sum_{m=j+1}^{N-1} a_{m}\left(\begin{array}{c}
m \\
j
\end{array}\right) j !\left(\begin{array}{l}
p \\
j
\end{array}\right)(m-j) x^{m-j-1} u_{0}^{p-j}\right] \\
= & \quad\left[\sum_{j=0}^{p} \sum_{m=j}^{N-1} a_{m}\left(\begin{array}{c}
m \\
j
\end{array}\right) j !\left(\begin{array}{c}
p \\
j
\end{array}\right) x^{m-j} u_{0}^{p-j+1}\right. \\
& \left.+\sum_{j=0}^{p} \sum_{m=j+1}^{N-1} a_{m}\left(\begin{array}{c}
m \\
j
\end{array}\right) j !\left(\begin{array}{l}
p \\
j
\end{array}\right)(m-j) x^{m-j-1} u_{0}^{p-j}\right] .
\end{aligned}
$$


In the second sum, let $j_{1}=j+1$, so $-j=-j_{1}+1$. Then

$$
\begin{aligned}
\sum_{j=0}^{p} & \sum_{m=j+1}^{N-1} a_{m}\left(\begin{array}{c}
m \\
j
\end{array}\right) j !\left(\begin{array}{c}
p \\
j
\end{array}\right)(m-j) x^{m-j-1} u_{0}^{p-j} \\
\quad= & \sum_{j_{1}=1}^{p+1} \sum_{m=j_{1}}^{N-1} a_{m}\left(\begin{array}{c}
m \\
j_{1}-1
\end{array}\right)\left(j_{1}-1\right) !\left(\begin{array}{c}
p \\
j_{1}-1
\end{array}\right)\left(m-j_{1}+1\right) x^{m-j_{1}} u_{0}^{p+1-j_{1}} .
\end{aligned}
$$

Thus equation $(*)$ becomes

$$
\begin{aligned}
e^{u_{0} x}\left[\sum_{j=0}^{0} \sum_{m=j}^{N-1} a_{m}\left(\begin{array}{c}
m \\
j
\end{array}\right) j !\left(\begin{array}{c}
p+1 \\
j
\end{array}\right) x^{m-j} u_{0}^{p+1-j}\right. \\
+\sum_{j=1}^{p} \sum_{m=j}^{N-1} a_{m} x^{m-j} u_{0}^{p+1-j} \\
\quad \cdot\left\{\left(\begin{array}{c}
m \\
j
\end{array}\right) j !\left(\begin{array}{l}
p \\
j
\end{array}\right)+\left(\begin{array}{c}
m \\
j-1
\end{array}\right)(j-1) !\left(\begin{array}{c}
p \\
j-1
\end{array}\right)(m-j+1)\right\} \\
\left.+\sum_{m=j}^{N-1} a_{m}\left(\begin{array}{c}
m \\
p+1-1
\end{array}\right)(p+1-1) !\left(\begin{array}{c}
p \\
p+1-1
\end{array}\right)(m-p-1+1) x^{m-p-1}\right]
\end{aligned}
$$

But

$$
\left(\begin{array}{c}
m \\
j
\end{array}\right) j !\left(\begin{array}{c}
p \\
j
\end{array}\right)+\left(\begin{array}{c}
m \\
j-1
\end{array}\right)(j-1) !\left(\begin{array}{c}
p \\
j-1
\end{array}\right)(m-j+1)=\left(\begin{array}{c}
m \\
j
\end{array}\right) j !\left(\begin{array}{c}
p+1 \\
j
\end{array}\right)
$$

so this becomes

$$
e^{u} 0^{x}\left[\sum_{j=0}^{p+1} \sum_{m=j}^{N-1} a_{m}\left(\begin{array}{c}
m \\
j
\end{array}\right) j !\left(\begin{array}{c}
p+1 \\
j
\end{array}\right) x^{m-j} u_{0}^{p+1-j}\right]
$$

which completes the proof of the claim.

Now using the claim, we have

$$
\int_{-\infty}^{+\infty} f(x-t) d \mu(t)=\sum_{n=0}^{K} b_{n} \frac{d^{n}}{d x^{n}}[f(x)] \quad \text { for } f(x)=\sum_{m=0}^{N-1} a_{m} x^{m} e^{u_{0} x}
$$

as desired.

We can extend the previous proposition to the infinite case of the theorem, i.e.,

Proposition 2.5. If $z=u_{0}$ is a zero of multiplicity $N$ of the function

$$
g(z)=\sum_{n=0}^{\infty} b_{n} z^{n}-\int e^{-z t} d \mu(t),
$$

where $\Sigma_{n=0}^{\infty} b_{n} z^{n}$ is analytic in $|z| \leqslant a$, and $\left|u_{0}\right| \leqslant a$, then $f(x)=P(x) e^{u 0^{x}}$, 
where $P(x)$ is a polynomial of degree $N-1$, is a solution of the equation $\Sigma_{n=0}^{\infty} b_{n} f^{(n)}(x)=\int f(x-t) d \mu(t)$.

Proof. Since $\Sigma_{n=0}^{\infty} b_{n} z^{n}$ is analytic for $|z| \leqslant a$, the hypothesis that $u_{0}$ is contained in this disc and is an $N$-fold zero of (7) allows us to differentiate the series term by term to obtain

$$
\int(-t)^{j} e^{-u_{0} t} d \mu(t)=\sum_{n=j}^{\infty} j !\left(\begin{array}{l}
n \\
j
\end{array}\right) b_{n} u_{0}^{n-j} \text { for } 0 \leqslant j \leqslant N-1 .
$$

Working as before, we obtain

$$
\int f(x-t) d \mu(t)=e^{u 0^{x}} \sum_{m=0}^{N-1} \sum_{j=0}^{m} \sum_{n=j}^{\infty} a_{m}\left(\begin{array}{c}
m \\
j
\end{array}\right) j !\left(\begin{array}{l}
n \\
j
\end{array}\right) b_{n} x^{m-j} u_{0}^{n-j}
$$

where $f(x)$ is defined as above with $P(x)=\Sigma_{m=0}^{N-1} a_{m} x^{m}$. Since the infinite series converges uniformly for $z=u_{0}$ since $\left|u_{0}\right| \leqslant a$, we may change the order of summation to obtain

$$
\begin{aligned}
\int f(x-t) d \mu(t) & =e^{u_{0} x} \sum_{n=0}^{\infty} \sum_{j=0}^{n} \sum_{m=j}^{N-1} a_{m}\left(\begin{array}{c}
m \\
j
\end{array}\right) j !\left(\begin{array}{l}
n \\
j
\end{array}\right) b_{n} x^{m-j} u_{0}^{n-j} \\
& =\sum_{n=0}^{\infty} b_{n} f^{(n)}(x)
\end{aligned}
$$

as in the previous proposition.

REMARK. Including an extra finite sum in the above proof will show that any combination of the above types of functions, i.e., $\Sigma_{n=0}^{N} c_{n} P_{n}(x) e^{\alpha} n^{x}$, will also be a solution. Proposition 2.6 will show that such sums are the only such solutions which are entire of exponential type, while Propositions 3.4 and 3.5 concern analyticity of solutions of exponential growth on the real line.

EXAMPLES. (I) Let

$$
d \mu(t)= \begin{cases}d t & \text { if } 0<t<1 \\ 0 & \text { otherwise }\end{cases}
$$

To construct solutions of the equation

$$
f^{\prime}(x)=\int_{0}^{1} f(x-t) d t
$$

we look at the roots of the function

$$
g(z)=\int e^{-z t} d \mu(t)-z=\frac{1-e^{-z}}{z}-z,
$$

i.e., the complex numbers such that $e^{-z}=1-z^{2}$ (for $z \neq 0$ ). Since there are an infinite number of roots of this equation, there are an infinite number of solutions of (8). 
Note. It will follow from Theorem 3.2 that if $f(x)$ is bounded on the real line and satisfies (8), then $f(x)$ is entire of exponential type 1 . Thus there are no roots of $e^{-z}=1-z^{2}$ of the form $z=i y$ for $|y|>1$.

(II) Let

$$
d \mu(t)= \begin{cases}d t & \text { for } t>0 \\ 0 & \text { otherwise }\end{cases}
$$

To construct solutions of the equation

$$
f^{\prime}(x)=\int_{0}^{+\infty} f(x-t) d t
$$

consider the roots of

$$
g(z)=z-\int_{0}^{\infty} e^{-z t} d t=z-\frac{1}{z} \quad(\operatorname{Re} z>0) .
$$

Thus $z=1 / z$ is the equation to be satisfied for $\operatorname{Re} z>0$, so that the root is $z=+1$. Thus solutions we can construct are of the form $f(x)=c e^{x}$, for constant $c$.

Conversely, since $\int_{0}^{\infty} e^{-t} d t=1$, if $|f(x)| \leqslant M e^{x}$ for some constant $M$ and all real $x$, and $f(x)$ satisfies (9), Lemma 1 will show that $\left|f^{(n)}(x)\right| \leqslant M e^{x}$ for $n \geqslant 1$ also. A theorem of Tagamlizki [21] then says $f(x)=M e^{x}$.

Conclusion. Any solution of (9) which is of exponential growth on the real line is of the form $f(x)=c e^{x}$. Thus, solutions of (9) have a remarkable resemblance to solutions of $f^{\prime}(x)=f(x)$.

(III) Consider the related equation

$$
f^{\prime}(x)-4 f(x)=\int_{0}^{\infty}-4 f(x-t) d t
$$

Since $z-4+4 / z=0 \Longleftrightarrow(z-2)^{2}=0, z=2$ is a root with multiplicity 2. Thus $\left(c_{1} x+c_{2}\right) e^{2 x}$ is a solution of (10) for arbitrary complex constants $c_{1}$ and $c_{2}$.

We now show that every solution of equation (1) which is entire of exponential type is a sum of exponential functions (Proposition 2.5 shows that such a sum is a solution). H. R. Pitt [17] has investigated solutions of equations of the form $\Sigma_{r=0}^{R} \int f^{(r)}(x-y) d k_{r}(y)=0$, where the $k_{r}(y)$ are functions of bounded variation in any finite interval. In Proposition 3.4, we show that every $C^{n}(R)$ solution of $f^{(n)}(x)=\int f(x-t) d \mu(t)$ which is of exponential growth on the real line is actually entire of exponential type. In Proposition 3.5 we are able to show that every $C^{k}(R)$ solution of (5) which is of exponential growth on the real line is actually infinitely differentiable. Thus it is reasonable at the start to make the assumption that the solution is entire of exponential type. These results are similar to those of Pitt, but the proof is new and applies to the case of the differential equation of infinite order. 
Proposition 2.6. Let $f(z)$ be entire of exponential type $a, \Sigma_{n=0}^{\infty} a_{n} z^{n}$ be analytic in a disc $|z| \leqslant b$ which contains the conjugate indicator diagram of $f(z)$, $\left|\int e^{-z t} d \mu(t)\right|<\infty$ for $|\operatorname{Re} z| \leqslant c$, where $c \geqslant b$, and

$$
\sum_{n=0}^{\infty} a_{n} f^{(n)}(x)=\int f(x-t) d \mu(t) .
$$

Then $f(z)=\Sigma_{k=0}^{M} P_{k}(z) e^{\alpha} k^{z}$, where the $\alpha_{k}$ are elements of the set of zeros (with multiplicity $m_{k}$ ) of the function

$$
g(z)=\int e^{-z t} d \mu(t)-\sum_{n=0}^{\infty} a_{n} z^{n}
$$

which are contained in the conjugate indicator diagram of $f(z)$, the $P_{k}(z)$ are polynomials depending on $f(z)$ of degree $m_{k}-1$, and $M$ is possibly infinite.

Note. We assume that $g(z)$ is not identically zero.

Proof. Let $C$ be a contour which contains the conjugate indicator diagram of $f(z)$ in its interior, and such that $C$ is contained in the disc $|z| \leqslant b$. It is well known that $C$ can always be chosen to be the circle $|z|=a+\epsilon, \epsilon>0$. Then $f(z)=\int_{C} e^{z \omega} \varphi(\omega) d \omega$, where $\varphi(\omega)$ is the Borel transform of $f(z)$. Then since $f(z)$ satisfies the equation above,

$$
\begin{aligned}
& \sum_{0}^{\infty} a_{n} \int_{C} \omega^{n} e^{z \omega} \varphi(\omega) d \omega=\iint_{C} e^{(z-t) \omega} \varphi(\omega) d \omega d \mu(t) \\
& \Rightarrow \int_{C} \sum_{0}^{\infty} a_{n} \omega^{n} \cdot e^{z \omega} \varphi(\omega) d \omega=\int_{C} \int e^{z \omega} e^{-t \omega} \varphi(\omega) d \mu(t) d \omega \\
&=\int_{C} e^{z \omega}\left\{\int e^{-t \omega} d \mu(t)\right\} \varphi(\omega) d \omega \\
& \Rightarrow \int_{C} e^{z \omega}\left\{\sum_{0}^{\infty} a_{n} \omega^{n}-\int e^{-t \omega} d \mu(t)\right\} \varphi(\omega) d \omega=0 .
\end{aligned}
$$

We assume that $\Sigma_{n=0}^{\infty} a_{n} \omega^{n}-\int e^{-t \omega} d \mu(t)$ is not identically zero. Since $\varphi(\omega) \cdot$ $\left\{\Sigma_{n=0}^{\infty} a_{n} \omega^{n}-\int e^{-t \omega} d \mu(t)\right\}$ is regular on $C$, it is analytic inside $C$ by Pólya [1, p. 110].

Then $\varphi(\omega)$ has (at most) poles at the zeros of $\Sigma_{n=0}^{\infty} a_{n} \omega^{n}-\int e^{-t \omega} d \mu(t)$. Each pole of multiplicity $m$ of $\varphi(\omega)$ contributes a factor of the form $P(z) e^{\alpha z}$ to $f(z)$, where $\alpha$ is the pole, and $P(z)$ is a polynomial of multiplicity $m-1$ depending on $\varphi(\omega)$. Since $\varphi(\omega)$ is analytic outside of the conjugate indicator diagram of $f(z)$, only values in this set could be poles of $\varphi(\omega)$.

The function $\Sigma_{n=0}^{\infty} a_{n} \omega^{n}-\int e^{-t \omega} d \mu(t)$ could have an infinite number of zeros in that set, and thus there could be an infinite number of poles.

REMARK. The previous proposition allows us to prove the classical theorem 
that an entire function of exponential type which is $2 \pi$ periodic on the real axis is an exponential polynomial.

For if $d \mu(t)$ is point mass at $-2 \pi$, we are considering the equation

$$
f(x)=f(x+2 \pi) \quad\left(a_{n}=0 \text { if } n \geqslant 1\right) .
$$

The set of zeros of $\int e^{-z t} d \mu(t)-1=e^{2 \pi z}-1$ are the points $z=i k, k=0, \pm 1$, $\pm 2, \ldots$, and the zeros are simple. Thus

$$
f(z)=\sum_{k=-n}^{n} d_{k} e^{i k z} \text { for }|n| \leqslant \operatorname{type} f .
$$

3. Solutions of equations of the form $\Sigma_{n=0}^{\infty} a_{n} f^{(n)}(x)=\int f(x-t) d \mu(t)$. A. Giroux [7] has shown that a bounded function $f(x)$ which satisfies the equation $f^{\prime}(x)=\int f(x-t) d \mu(t)$ for all real $x$, where $\mu$ is a complex measure, is an entire function of exponential type $|\mu|(R)$. Theorem 3.1 is an extension of this theorem to the case when the function is not necessarily bounded on the real axis.

The remainder of this section deals with equations of the above form when the measure is complex but does not necessarily have a finite Laplace transform on any interval of the real line; these results cannot be obtained from those in $\$ 2$. First, in Theorem 3.2, we consider the cases when $f(x)$ is a bounded function for real $x$ and

$$
f^{(n)}(x)=\int f(x-t) d \mu(t) \text { for } n \geqslant 2,
$$

and then, in Theorem 3.3, consider the same type of function when the left side of the equation is of the form $P(D)$, where $D$ is differentiation and $P(z)$ is a polynomial.

Finally, we consider these same equations in the same order but for the case that the function may have exponential growth on the real line and the measure has a finite bilateral Laplace transform in an interval about the origin. We have already shown in Proposition 2.2 that solutions of the differential equation of infinite order are entire of exponential type, and the Propositions 3.4 and 3.5 following are concerned with those equations with the differential operator of finite order. Recall also Proposition 2.6 for the particular form of the solutions in the case that they are entire functions of exponential type.

To consider several cases at the same time in the first theorem, we make the following definition:

Definition. Let $f(x)$ be continuous for all real $x$. We say that $\psi(x) \in$ $C(R)$ is a bounding function for $f(x)$ if

(i) $|f(x)| \leqslant \psi(x)$ for all real $x$ and

(ii) $\exists A_{0}(\psi) \ni \psi(x-t) \leqslant A_{0} \psi(x) \psi(-t)$ for all $x$ and $t$. 
The smallest such constant, denoted $A$, will be called the supporting constant.

ExAMPLES. (a) $\psi(x)=e^{a|x|}$, since $e^{a|x-t|} \leqslant e^{a|x|} e^{a|t|} . A=1$.

(b) $\psi(x)=e^{a x}$, since $\exp (a(x-t)) \leqslant \exp (a x) \exp (-a t) . A=1$.

(c) $\psi(x)=M>0$, because $M=A M M . A=1 / M$.

LEMMA 1. Let $f \in C^{1}(R), \psi$ be a bounding function for $f^{\prime}$, and define $h(x)=\int f(x-t) d \mu(t)$. If $\int \psi(-t) d|\mu|(t)<\infty$, then

$$
h^{\prime}(x)=\int f^{\prime}(x-t) d \mu(t)
$$

Proof.

$$
\begin{aligned}
h^{\prime}(x) & =\lim _{a \rightarrow 0} \frac{h(x+a)-h(x)}{a}=\lim _{a \rightarrow 0} a^{-1} \int[f(x+a-t)-f(x-t)] d \mu(t) \\
& =\lim _{a \rightarrow 0} \int f^{\prime}(x+a \theta-t) d \mu(t) \text { for } 0<\theta<1
\end{aligned}
$$

by the mean value theorem. But $\left|f^{\prime}(x+a \theta-t)\right| \leqslant A \cdot \psi(x+a \theta) \cdot \psi(-t) \in$ $L^{1}(d|\mu|(t))$. Thus an application of the dominated convergence theorem allows us to conclude that

$$
h^{\prime}(x)=\int \lim _{a \rightarrow 0} f^{\prime}(x+a \theta-t) d \mu(t)=\int f^{\prime}(x-t) d \mu(t) .
$$

LEMMA 2. If $\left|f^{(n)}(x)\right| \leqslant B^{n} \psi(x)$ for $n \geqslant 0$ and $x$ real, where $\psi(x) \in C(R)$, then $f$ is entire of exponential type $B$.

Proof. We first show that $f(x)$ is represented by its Taylor series about zero. If $R_{n}$ is the remainder term in the series, then

$$
R_{n-1}(x)=\frac{f^{(n)}(\xi) x^{n}}{n !} \text { for some }|x|>|\xi|>0 .
$$

But

$$
\left|R_{n-1}(x)\right| \leqslant \frac{B^{n} \psi(\xi)|x|^{n}}{n !}=\psi(\xi) \frac{|B x|^{n}}{n !} \text { for every } n
$$

and the latter term vanishes as $n \rightarrow \infty$. So $f(x)$ is represented by its Taylor series for any $x$.

Define $f(z)=\Sigma_{n=0}^{\infty} f^{(n)}(0) z^{n} / n$ !. Then $f(x)$ is the original function for real $x$. This series defines an entire function, since the radius of convergence of the series is

$$
\left[\limsup _{n \rightarrow \infty}\left|\frac{f^{(n)}(0)}{n !}\right|^{1 / n}\right]^{-1} \geqslant\left[\lim _{n \rightarrow \infty} B\left[\frac{\psi(0)}{n !}\right]^{1 / n}\right]^{-1}=\infty .
$$

Further, we have 


$$
\begin{aligned}
\limsup _{n \rightarrow \infty}\left|n ! \frac{f^{(n)}(0)}{n !}\right|^{1 / n} & =\limsup _{n \rightarrow \infty}\left|f^{(n)}(0)\right|^{1 / n} \\
& \leqslant \lim _{n \rightarrow \infty}\left|B^{n} \psi(0)\right|^{1 / n}=B,
\end{aligned}
$$

so the function is entire of exponential type $B$.

THEOREM 3.1. Suppose $f \in C(R)$ has the bounding function $\psi(x)$. Let $V=$ $\int \psi(-t) d|\mu|(t)<\infty$ for some complex measure $\mu(t)$. If for each real $x$

$$
f^{\prime}(x)=\int f(x-t) d \mu(t)
$$

then $f$ is an entire function of exponential type $A V, A$ the supporting constant.

Proof. Fix $a$, and choose a sequence such that $x_{n} \rightarrow a$ as $n \rightarrow \infty$. Then for $\delta>0$,

$$
\lim _{x_{n \rightarrow a}}\left[f^{\prime}\left(x_{n}\right)-f^{\prime}(a)\right]=\lim _{n \rightarrow \infty} \int\left[f\left(x_{n}-t\right)-f(a-t)\right] d \mu(t) .
$$

But

$$
\begin{aligned}
\left|f\left(x_{n}-t\right)-f(a-t)\right| \leqslant \psi\left(x_{n}-t\right)+\psi(a-t)=\left\{\psi\left(x_{n}\right)+\psi(a)\right\} \psi(-t) \\
\leqslant \underset{+a-\delta \leqslant x \leqslant a+\delta}{2 \max |\psi(x)| \cdot \psi(-t) \in L^{1}(d|\mu|(t))}
\end{aligned}
$$

so that the dominated convergence theorem implies

$$
\lim _{n \rightarrow \infty}\left[f^{\prime}\left(x_{n}\right)-f^{\prime}(a)\right]=\int \lim _{n \rightarrow \infty}\left[f\left(x_{n}-t\right)-f(a-t)\right] d \mu(t)=0
$$

for every such sequence. Hence $f^{\prime} \in C(R)$.

Since

$$
\begin{aligned}
\left|f^{\prime}(x)\right| & \leqslant \int|f(x-t)| d|\mu|(t) \\
& \leqslant A \psi(x) \int \psi(-t) d|\mu|(t)=A V \psi(x),
\end{aligned}
$$

we see that $f^{\prime}(x)$ has the bounding function $A V \cdot \psi(x)$. By Lemma $1, f^{(2)}(x)=$ $\int f^{\prime}(x-t) d \mu(t) ;$ so

$$
\left|f^{(2)}(x)\right| \leqslant A V \int \psi(x-t) d|\mu|(t) \leqslant(A V)^{2} \psi(x) .
$$

By reiterating this procedure, we see that $f \in C^{\infty}(R)$,

$$
f^{(n)}(x)=\int f^{(n-1)}(x-t) d \mu(t), \text { for } n \geqslant 1,
$$

and

$$
\left|f^{(n)}(x)\right| \leqslant(A V)^{n} \psi(x) \text { for } n \geqslant 0
$$


The conclusion follows from Lemma 2.

EXAMPLES. (1) If $f(x)$ is bounded on the real axis and (11) holds, then $|f(x)| \leqslant M=\psi(x)$ has supporting constant $A=1 / M$. Thus, the type of $f$ is given by

$$
\frac{1}{M} \int_{R} M d|\mu|=|\mu|(R)
$$

This is just Giroux's theorem [7].

(2) Let $f(x)=e^{2 x}=\psi(x)$, and let $d \mu(t)$ be the measure which has mass 2 at $t=0$. Then (11) holds, and the type of $f$ is given by

$$
V=\int e^{-2 t} d|\mu|(t)=2 .
$$

(3) Let $-\infty<t<+\infty$ and

$$
d \mu(t)=\left\{\begin{array}{l}
\tau \frac{4(-1)^{k}}{\pi^{2}(2 k+1)^{2}} \\
0 \text { otherwise. }
\end{array}\right.
$$

Boas [1] has shown that

$$
f^{\prime}(x)=\int f(x-t) d \mu(t)=\frac{4 \tau}{\pi^{2}} \sum_{k=-\infty}^{+\infty} \frac{(-1)^{k}}{(2 k+1)^{2}} f\left(x+\frac{2 k+1}{2 \tau} \pi\right)
$$

for every entire function of exponential type $\tau$ which is bounded on the real axis. In this case

$$
|\mu|(R)=\frac{4 \tau}{\pi^{2}} \sum_{-\infty}^{+\infty} \frac{1}{(2 k+1)^{2}}=\tau,
$$

so that the converse also holds.

We can extend this theorem to more general equations. The growth of the function $f(x)$ along the real line determines the form of proof required, and we first present the cases where $f(x)$ is bounded for real $x$ before moving on to a more general situation.

THEOREM 3.2. Suppose that $f \in C^{n-1}(R), n \geqslant 1,|f(x)| \leqslant M$ for real $x$, and there is a complex measure $\mu$ such that

$$
f^{(n)}(x)=\int f(x-t) d \mu(t)
$$

Then $f$ is entire of exponential type $[|\mu|(R)]^{1 / n}$ for $n \geqslant 1$.

Proof. If $n=1$, the theorem follows from Theorem 3.1 for the case when $f(x)$ is bounded. Thus we assume $n \geqslant 2$. Let $K=|\mu|(R)$. It follows from (12) that

$$
\left|f^{(n)}(x)\right| \leqslant \int|f(x-t)| d|\mu|(t) \leqslant M K
$$


If $M_{j}=\max _{x}\left|f^{(j)}(x)\right|$ for $j \geqslant 1$, then Kolmogorov's inequality [11] states

$$
\left|f^{(k)}(x)\right| \leqslant \gamma_{n, k^{M}} M^{1-k / n} M_{n}^{k / n} \text { for } 0<k \leqslant n .
$$

The constants $\gamma_{n, k}$ are well known and are such that $1<\gamma_{n, k}<\pi / 2$ for $0<k<$ $n$. Thus we have

$$
\left|f^{(k)}(x)\right| \leqslant \gamma_{n, k} M^{1-k / n}(M K)^{k / n}=\gamma_{n, k} K^{k / n} M^{(n-k+k) / n} \quad \text { for } 0<k \leqslant n .
$$

Let $C=\max _{1 \leqslant k \leqslant n}\left\{\gamma_{n, k}\right\}$. In general, then $\left|f^{(k)}(x)\right| \leqslant C K^{k / n} M$ for $0<k \leqslant n$.

It follows as in the proof of the previous proposition that $f \in C^{n}(R)$. The constant $M K$ is a bounding function for $f^{(n)}(x)$, so Lemma 1 gives $f^{(n+1)}(x)=$ $\int f^{\prime}(x-t) d \mu(t)$. But then

$$
\left|f^{(n+1)}(x)\right| \leqslant \int\left|f^{\prime}(x-t)\right| d|\mu|(t) \leqslant C K^{1 / n} M \cdot K=C K^{(n+1) / n} M .
$$

So again we may show $f \in C^{n+1}(R)$, and Lemma 1 gives $f^{(n+2)}(x)=$ $\int f^{(2)}(x-t) d \mu(t)$. Iteration of this procedure $n$ times gives

$$
f^{(n+k)}(x)=\int f^{(k)}(x-t) d \mu(t) \text { for } 0 \leqslant k \leqslant n
$$

and

$$
\left|f^{(n+k)}(x)\right| \leqslant C K^{n+k / n} M \text { for } 0 \leqslant k \leqslant n .
$$

But then we may repeat this procedure to show that

$$
f^{(n+j)}(x)=\int f^{(j)}(x-t) d \mu(t) \text { for } j \geqslant 0
$$

and obtain

$$
\left|f^{(i)}(x)\right| \leqslant C K^{i / n} M \quad \text { for } i \geqslant 0 .
$$

Now Lemma 2 applies, with $B^{i}=\left(K^{1 / n}\right)^{i}$ for $i \geqslant 0$ and $\psi(x)=C M$. That is, $f$ is entire of exponential type $K^{1 / n}$.

ExAmples. (1) Let $d \mu(t)$ have mass -9 at $t=0$. Then for $n=2,(12)$ becomes $f^{(2)}(x)=-9 f(x)$ which has the function $\sin (3 x)$ as a solution. Note that $\sin (3 x)$ is entire of exponential type $(|\mu|(R))^{1 / 2}=(|-9|)^{1 / 2}=3$.

(2) Let $d \mu(t)$ have mass -1 at $t=\pi / 2$. If $n=5$, (12) becomes the differential-difference equation $f^{(5)}(x)=-f(x-\pi / 2)$. Since $\cos (x-\pi / 2)=\sin x$ and $d^{5}[\cos (x)] / d x^{5}=-\sin x$, we see that $\cos x$ is a solution of this equation.

This agrees with the theorem since $\cos x$ is entire of exponential type $(|-1|)^{1 / 5}=1$.

The final theorem dealing specifically with functions which are bounded on the real line for this section is the following:

TheOREM 3.3. Let $f \in C^{n}(R),|f(x)| \leqslant M$ for real $x$. Suppose 


$$
L[f(x)]=\sum_{i=0}^{n} c_{i} f^{(i)}(x)=\int f(x-t) d \mu(t),
$$

where $\mu$ is a complex measure and the coefficients satisfy $\left|c_{i}\right| \leqslant C^{i}$ for $i=0,1$, $\ldots, n-1$ and $\left|c_{n}\right|=C^{n}$ for some constant $C$.

Then $f$ is entire of exponential type $2^{(n-1) / n} C^{-1}\left[\gamma^{n}+V\right]^{1 / n}$, where $V$ is here the variation of the measure $|\mu|(R)$, and $\gamma=\Sigma_{k=1}^{n}\left\{\gamma_{n, n-k}\right\}^{1 / k}$ where the $\boldsymbol{\gamma}_{n, k}$ are those constants determined by Kolmogorov which solve Landau's problem for $L^{\infty}(R)$ (as on $p .161$ ).

Proof. Let $L_{1}[f(x)]=C^{-n} L[f(x)]$. Since $L_{1}[f(x)]=C^{-n} \int f(x-t) d \mu(t)$,

$$
\left|L_{1}[f(x)]\right| \leqslant C^{-n} M V=M V_{1} \text { if } V_{1}=C^{-n} V .
$$

Since $L_{1}[f(x)]$ and $f(x)$ are both bounded on the real line, we may apply Landau's Theorem 1 from [13] to say that $\left|f^{(k)}(x)\right|$ is bounded for $0<k \leqslant n$.

In particular, $\left|f^{(n)}(x)\right|$ is bounded, so we may again apply Kolmogorov's theorem to obtain precise bounds on the intermediate derivatives: $\left|f^{(k)}(x)\right| \leqslant$ $M_{k} \leqslant \gamma_{n, k^{M}} M^{(n-k) / n} M_{n}^{k / n}, 0 \leqslant k \leqslant n$. Equality does hold for the case of the spline functions, as Schoenberg [19] shows. Now,

$$
f^{(n)}(x)=L_{1}[f(x)]-\sum_{i=0}^{n-1} d_{i} f^{(i)}(x),
$$

where $\left|d_{i}\right| \leqslant C^{i-n}=\left(C^{-1}\right)^{n-i}$. Let $D=C^{-1}$, so that $\left|d_{i}\right| \leqslant D^{n-i}$ for $i=0,1$, $\ldots, n-1$. Thus

$$
M_{n} \leqslant M V_{1}+\sum_{k=0}^{n-1} D^{n-k} M_{k} \leqslant M V_{1}+\sum_{k=0}^{n-1} D^{n-k} \gamma_{n, k} M^{(n-k) / n} M_{n}^{k / n}
$$

Now we apply Lemma 3 of Halperin and Pitt [8] with $x_{n}=M_{n}^{1 / n}, a_{n}=M V_{1}+$ $D^{n} \gamma_{n, 0} M, \ldots, a_{0}=D \gamma_{n, n-1} M^{1 / n}$ to obtain

$$
\begin{aligned}
M_{n}^{1 / n} \leqslant & D \gamma_{n, n-1} M^{1 / n}+\left[D^{2} \gamma_{n, n-2} M^{2 / n}\right]^{1 / 2} \\
& +\cdots+\left[D^{n-1} \gamma_{n, 1} M^{(n-1) / n}\right]^{1 /(n-1)}+\left[M V_{1}+D^{n} \gamma_{n, 0} M\right]^{1 / n} \\
\Rightarrow & M_{n}^{1 / n} \leqslant D \gamma_{n, n-1} M^{1 / n}+D M^{1 / n}\left[\gamma_{n, n-2}\right]^{1 / 2} \\
& +\cdots+D M^{1 / n}\left[\gamma_{n, 1}\right]^{1 /(n-1)}+\left\{\left(M V_{1}\right)^{1 / n}+D M^{1 / n} \gamma_{n, 0}^{1 / n}\right\}
\end{aligned}
$$

where we used the inequality $(a+b)^{1 / n} \leqslant a^{1 / n}+b^{1 / n}$, for $a, b \geqslant 0$.

$$
\Rightarrow M_{n}^{1 / n} \leqslant D M^{1 / n}\left\{\sum_{k=1}^{n}\left\{\gamma_{n, n-k}\right\}^{1 / k}\right\}+M^{1 / n} V_{1}^{1 / n}
$$

or if $\gamma=\Sigma_{k=1}^{n}\left\{\gamma_{n, n-k}\right\}^{1 / k}$,

$$
\Rightarrow M_{n}^{1 / n} \leqslant D M^{1 / n} \gamma+M^{1 / n} V_{1}^{1 / n} \text {. }
$$


So using Kolmogorov's inequality again

$$
\begin{aligned}
M_{k} & \leqslant \gamma_{n, k} M^{(n-k) / n} M_{n}^{k / n} \\
& \leqslant \gamma_{n, k} M^{(n-k) / n}\left[D M^{1 / n} \gamma+M^{1 / n} V_{1}^{1 / n}\right]^{k}, \quad 0 \leqslant k \leqslant n, \\
& \Rightarrow M_{k} \leqslant \gamma_{n, k^{2}} M^{(n-k) / n} 2^{k-1}\left[D^{k} M^{k / n} \gamma^{k}+M^{k / n} V_{1}^{k / n}\right]
\end{aligned}
$$

where we used the inequality $(a+b)^{k} \leqslant 2^{k-1}\left(a^{k}+b^{k}\right)$ for $a, b \geqslant 0$. Since $V_{1}=D^{n} V$,

$$
M_{k} \leqslant \gamma_{n, k^{M}} M^{(n-k) / n} 2^{k-1} M^{k / n} D^{k}\left[\gamma^{k}+V^{k / n}\right]
$$

so the general inequality is

$$
M_{k} \leqslant \gamma_{n, k} 2^{k-1} M D^{k}\left[\gamma^{k}+V^{k / n}\right], \quad 0 \leqslant k \leqslant n .
$$

In particular, $M_{n} \leqslant 2^{n-1} M D^{n}\left[\gamma^{n}+V\right]$, since $\gamma_{n, n}=1$.

EXAMPLE. If $n=2$, we have $M_{1} \leqslant \sqrt{2} M C^{-1}\left[(1+\sqrt{2})+V^{1 / 2}\right]$ and $M_{2} \leqslant 2 M C^{-2}\left[(1+\sqrt{2})^{2}+V\right]$.

Now note that

$$
\frac{d}{d x}\left(\sum_{i=0}^{n} c_{i} f^{(i)}(x)\right)=\int f^{\prime}(x-t) d \mu(t)
$$

by Lemma 1. I.e.,

$$
\sum_{i=0}^{n} c_{i} f^{(i+1)}(x)=\int f^{\prime}(x-t) d \mu(t),
$$

which says that $f^{\prime}(x)$ satisfies (13), and $f \in C^{n+1}(R)$. Iterating this procedure, we see that $f^{(k)}(x)$ satisfies (13) for $0 \leqslant k \leqslant n$, and that $f \in C^{2 n}(R)$.

If we then apply the inequalities (14) to the function $f^{(k)}(x)$ for $k$ fixed, $0<k \leqslant n$, we obtain

$$
\begin{aligned}
M_{k+j} & \leqslant \gamma_{n, j} 2^{j-1} M_{k} D^{j}\left[\gamma^{j}+V^{j / n}\right], \quad 0 \leqslant j \leqslant n, \\
& \Rightarrow M_{k+j} \leqslant \gamma_{n, j} 2^{j-1}\left\{\gamma_{n, k} 2^{k-1} D^{k} M\left[\gamma^{k}+V^{k / n}\right]\right\} D^{j}\left[\gamma^{j}+V^{j / n}\right] \\
& \Rightarrow M_{k+j} \leqslant \gamma_{n, j} \gamma_{n, k} 2^{(j+k)-2} D^{k+j} M\left[\gamma^{k}+V^{k / n}\right]\left[\gamma^{j}+V^{j / n}\right] .
\end{aligned}
$$

In particular $M_{2 n} \leqslant 2^{2 n-2} D^{2 n} M\left[\gamma^{n}+V\right]^{2}$. By repeating this procedure on each successive block of $n$ numbers, we obtain $f \in C^{\infty}(R)$, and $M_{j n} \leqslant 2^{j n-i} D^{i n} M\left[\gamma^{n}+V\right]^{j}$ for $j \geqslant 1$, with the other appropriate bounds on the intermediate derivatives.

We proceed as in Lemma 2 to define the entire function whose restriction to the real axis is $f(x)$. Since the exponential type is given by $\lim \sup _{k \rightarrow \infty} M_{k}^{1 / k}$, we first note that the lim sup will be attained by the subsequence of the $M_{k}$ 's such that $k$ is a multiple of $n$. That is, the exponential type is at most 


$$
\begin{aligned}
\lim _{j \rightarrow \infty} M_{j n}^{1 / j n} & \leqslant \lim _{j \rightarrow \infty}\left[2^{j n-j} D^{j n} M\left[\gamma^{n}+V\right]^{j}\right]^{1 / j n} \\
\Rightarrow \lim _{j \rightarrow \infty} M_{j n}^{1 / j n} & \leqslant \lim _{j \rightarrow \infty} 2^{j(n-1) / j n} D M^{1 / j n}\left[\gamma^{n}+V\right]^{j / j n} \\
& =2^{(n-1) / n} C^{-1}\left[\gamma^{n}+V\right]^{1 / n}
\end{aligned}
$$

as claimed.

EXAmple. $f(x)=e^{i x}$ is a solution of the equation

$$
-4 f^{\prime \prime}(x)+2 i f^{\prime}(x)-f(x)=f(x)=\int f(x-t) d \mu(t)
$$

if $d \mu(t)$ is point mass at 0 . In this case, $C=2$, so the theorem gives

$$
\operatorname{type}\left(e^{i z}\right)=1 \leqslant 1 / 2 \sqrt{2}\left((1+\sqrt{2})^{2}+1\right)^{1 / 2} \sim 1.63 .
$$

We would like to show that similar theorems hold when $f(x)$ is exponentially bounded.

First,

LEMMA 3. If $\left|f^{(n)}(x)\right| \leqslant P e^{\tau|x|}$ for $\tau>0$ and $n \geqslant 1$, then

$$
\left|f^{(n-k)}(x)\right| \leqslant \tau^{-k}\left(P+C_{n-1} \tau+C_{n-2} \tau^{2}+\cdots+C_{n-k} \tau^{k}\right) e^{\tau|x|}
$$

where $C_{n-k}=\left|f^{(n-k)}(0)\right|, 0<k \leqslant n$.

Proof. (Induction on $k$ ). Suppose $k=1$. Since

$$
\begin{aligned}
& \int_{0}^{x} f^{(n)}(t) d t=f^{(n-1)}(x)-f^{(n-1)}(0) \\
& \quad \Rightarrow\left|f^{(n-1)}(x)\right| \leqslant\left|f^{(n-1)}(0)\right|+P \int_{0}^{x} e^{\tau|t|} d t=C_{n-1}+\left.\frac{1}{\tau} P e^{\tau|t|}\right|_{0} ^{x} \\
& \quad \Rightarrow\left|f^{(n-1)}(x)\right| \leqslant \tau^{-1}\left(P+C_{n-1}\right) e^{\tau|x|} .
\end{aligned}
$$

Now if $k>1$, we assume that

$$
\left|f^{(n-m)}(x)\right| \leqslant \tau^{-m}\left(P+C_{n-1} \tau+\cdots+C_{n-m} \tau^{m}\right) e^{\tau|x|} \text { for } 1 \leqslant m \leqslant k .
$$

Since

$$
\begin{aligned}
\left|f^{(n-k-1)}(x)-f^{(n-k-1)}(0)\right| \leqslant \int_{0}^{x}\left|f^{(n-k)}(t)\right| d t \\
\quad \leqslant \tau^{-k}\left(P+C_{n-1} \tau+\cdots+C_{n-k} \tau^{k}\right) \int_{0}^{x} e^{\tau|t|} d t \\
\Rightarrow\left|f^{(n-(k+1))}(x)\right| \\
\quad \leqslant\left|f^{(n-k-1)}(0)\right|+\tau^{-(k+1)}\left(P+C_{n-1} \tau+\cdots+C_{n-k} \tau^{k}\right) e^{\tau|x|} \\
\Rightarrow\left|f^{(n-(k+1))}(x)\right| \\
\quad \leqslant \tau^{-(k+1)}\left(P+C_{n-1} \tau+\cdots+C_{n-k} \tau^{k}+C_{n-(k+1)} \tau^{k+1}\right) e^{\tau|x|}
\end{aligned}
$$


In Proposition 2.6 we showed that an entire function of exponential type which satisfied an equation of the form in the following proposition was a sum of exponential functions. The following proposition makes it clear why this hypothesis on the analyticity of the function was chosen, since a solution which is of exponential growth on the real line is shown to be entire of exponential type.

Proposition 3.4. Let $f \in C^{n}(R),|f(x)| \leqslant M e^{\tau|x|}$ for $\tau>0$, and suppose

$$
f^{(n)}(x)=\int f(x-t) d \mu(t) \text { for } n \geqslant 2
$$

where $V=\int e^{\tau|t|} d|\mu|(t)<\infty$. Then $f$ is entire of exponential type at most $V^{1 / n}$.

Proof. We have

$$
\left|f^{(n)}(x)\right| \leqslant \int|f(x-t)| d|\mu|(t) \leqslant M e^{\tau|x|} \int e^{\tau|t|} d|\mu|(t)=M V e^{\tau|x|}
$$

By Lemma 3,

$$
\left|f^{(n-k)}(x)\right| \leqslant \tau^{-k}\left(M V+C_{n-1} \tau+\cdots+C_{n-k} \tau^{k}\right) e^{\tau|x|} \text { for } 0<k \leqslant n .
$$

By Lemma $1, f^{(n+1)}(x)=\int f^{\prime}(x-t) d \mu(t)$, and in fact $f^{(n+k)}(x)=$ $\int f^{(k)}(x-t) d \mu(t)$ for $0 \leqslant k \leqslant n$. Thus

$$
\left|f^{(n+k)}(x)\right| \leqslant \tau^{k-n}\left(M V+C_{n-1} \tau+C_{n-2} \tau^{2}+\cdots+C_{k} \tau^{n-k}\right) e^{\tau|x|} \cdot V
$$

for $0<k<n$, and $\left|f^{(2 n)}(x)\right| \leqslant M V^{2} e^{\tau|x|}$.

Repeating this process, we obtain $\left|f^{(m n+k)}(x)\right| \leqslant B_{k} V^{m} e^{\tau|x|}$ for $0<k<$ $n, m=1,2, \ldots$ where $B_{k}=\tau^{k-n}\left(M V+C_{n-1} \tau+C_{n-2} \tau^{2}+\cdots+C_{n} \tau^{n-k}\right)$ and $\left|f^{(m n)}(x)\right| \leqslant M V^{m} e^{\tau|x|}$ for $m=1,2, \ldots$.

Now,

$$
\limsup _{j \rightarrow \infty}\left|f^{(j)}(x)\right|^{1 / j} \leqslant \lim _{m \rightarrow \infty}\left[B_{k} V^{m}\right]^{(m n+k)^{-1}}=V^{1 / n}
$$

for $0<k<n$ fixed. $V^{1 / n}$.

It then follows from Lemma 2 that $f$ is entire of exponential type at most

REMARK. Proposition 2.3 showed that we could construct a solution of the equation $f^{(n)}(x)=\int f(x-t) d \mu(t)$ of the form $e^{\alpha z}$ if $z=\alpha$ was a zero of the function $g(z)=z^{n}-\int e^{-z t} d \mu(t)$, i.e., if $\alpha^{n}=\int e^{-\alpha t} d \mu(t)$. But Proposition 3.4 deals with the exponential type of solutions, and for functions $e^{\alpha z}$ of the above form we see that their exponential type $|\alpha|$ is such that

$$
|\alpha|=\left|\int e^{-\alpha t} d \mu(t)\right|^{1 / n} \leqslant\left\{\int\left|e^{-\alpha t}\right| d|\mu|(t)\right\}^{1 / n} \leqslant\left\{\int e^{-\operatorname{Re} \alpha \cdot t} d|\mu|(t)\right\}^{1 / n}
$$




$$
|\alpha| \leqslant\left\{\int e^{|\operatorname{Re} \alpha||t|} d|\mu|(t)\right\}^{1 / n}
$$

Since $\left|e^{\alpha x}\right| \leqslant e^{|\operatorname{Re} \alpha||x|}$ on the real line, the solutions which we can construct using Proposition 2.3 have exponential type agreeing with bounds given in Proposition 3.4 .

Further, a function of the form $e^{\alpha z}$ is bounded on the real line only for the case that $\alpha=i \beta, \beta$ real. For a solution of this form, the above analysis gives

$$
|i \beta| \leqslant\left\{\int\left|e^{-i \beta t}\right| d|\mu|(t)\right\}^{1 / n}=[|\mu|(R)]^{1 / n} .
$$

Thus again the exponential type of solutions which are bounded on the real line and which we can construct using Proposition 2.3 is that as predicted by Theorem 3.2 .

LEMmA 4. Let $f \in C^{N}(R)$, and $|f(x)| \leqslant M e^{\tau|x|}$. If $\left|\Sigma_{n=0}^{N} a_{n} f^{(n)}(x)\right| \leqslant$ $B e^{\tau|x|}$, then $\left|f^{(k)}(x)\right| \leqslant M_{k} e^{\tau|x|}, 0<k \leqslant N$, where $M_{k}$ is a constant depending upon the function and $k$, and $a_{N} \neq 0$.

PROOF. First we note that if $g(x)$ is a function such that $|g(x)| \leqslant M e^{\tau|x|}$ and if $h(x)=\int_{0}^{x} g(t) d t$, then $|h(x)| \leqslant \tau^{-1} M e^{\tau|x|}$.

(Induction on $N$.) Let $N=1$. Then

$$
\left|a_{1} f^{\prime}(x)+a_{0} f(x)\right| \leqslant B e^{\tau|x|} \Rightarrow\left|f^{\prime}(x)\right| \leqslant\left|a_{1}\right|^{-1}\left(B+\left|a_{0}\right| M\right) e^{\tau|x|}
$$

so that the lemma holds in this case.

Suppose that the lemma is true for $N=K$, and consider the case that $\left|\Sigma_{n=0}^{K+1} a_{n} f^{(n)}(x)\right| \leqslant B e^{\tau|x|}$.

If $h(x)=\int_{0}^{x}\left(\sum_{n=0}^{K+1} a_{n} f^{(n)}(t)\right) d t$, the note above gives $|h(x)| \leqslant \tau^{-1} B e^{\tau|x|}$. But

$$
\begin{aligned}
h(x) & =\sum_{n=0}^{K+1} a_{n} \int_{0}^{x} f^{(n)}(t) d t=\sum_{n=1}^{K+1} a_{n} \int_{0}^{x} f^{(n)}(t) d t+a_{0} \int_{0}^{x} f(t) d t \\
& =\sum_{n=0}^{K} a_{n+1} f^{(n)}(x)-\sum_{n=0}^{K} a_{n+1} f^{(n)}(0)+a_{0} \int_{0}^{x} f(t) d t .
\end{aligned}
$$

Then

$$
\begin{gathered}
\left|\sum_{n=0}^{K} a_{n+1} f^{(n)}(x)+a_{0} \int_{0}^{x} f(t) d t\right|-\left|\sum_{n=0}^{K} a_{n+1} f^{(n)}(0)\right| \leqslant|h(x)| \leqslant \tau^{-1} B e^{\tau|x|} \\
\Rightarrow\left|\sum_{n=0}^{K} a_{n+1} f^{(n)}(x)\right| \leqslant\left|a_{0}\right| \int_{0}^{x}|f(t)| d t+\left|\sum_{n=0}^{K} a_{n+1} f^{(n)}(0)\right|+\tau^{-1} B e^{\tau|x|} .
\end{gathered}
$$

Since $|f(x)| \leqslant M e^{\tau|x|}$, we use the above note again and let $p_{0}=\left|\sum_{n=0}^{K} a_{n+1} f^{(n)}(0)\right|$ so that 


$$
\left|\sum_{n=0}^{K} a_{n+1} f^{(n)}(x)\right| \leqslant\left(\left|a_{0}\right| M+p_{0}+\tau^{-1} B\right) e^{\tau|x|} .
$$

By the induction hypothesis, $\left|f^{(k)}(x)\right| \leqslant M_{k} e^{\tau|x|}$ for $0<k \leqslant K$. Further,

$$
\begin{aligned}
\left|f^{(K+1)}(x)\right| & \leqslant\left|a_{K+1}\right|^{-1}\left[\sum_{n=0}^{K}\left|a_{n}\right| M_{n} e^{\tau|x|}+B e^{\tau|x|}\right] \\
& \Rightarrow\left|f^{(K+1)}(x)\right| \leqslant\left|a_{K+1}\right|^{-1}\left\{\sum_{n=0}^{K}\left|a_{n}\right| M_{n}+B\right\} e^{\tau|x|}
\end{aligned}
$$

and the lemma is proved.

Proposition 3.5. Let $f \in C^{N}(R),|f(x)| \leqslant M e^{\tau|x|}$ for real $x$ and $\tau>0$, $a_{N} \neq 0$, and

$$
\sum_{n=0}^{N} a_{n} f^{(n)}(x)=\int f(x-t) d \mu(t),
$$

where $V=\int e^{\tau|t|} d|\mu|(t)<\infty$. Then $f(x)$ is infinitely differentiable.

Proof. Since

$$
\left|\sum_{n=0}^{N} a_{n} f^{(n)}(x)\right| \leqslant \int|f(x-t)| d|\mu|(t) \leqslant M e^{\tau|x|} \int e^{\tau|t|} d|\mu|(t)=V M e^{\tau|x|},
$$

Lemma 4 shows that $\left|f^{(k)}(x)\right| \leqslant M_{k} e^{\tau|x|}, 0<k \leqslant N$.

$$
\begin{aligned}
\sum_{n=0}^{N} a_{n} f^{(n)}(x) & =\int f(x-t) d \mu(t) \\
\Rightarrow f^{(N)}(x) & =\sum_{n=0}^{N-1}\left(-a_{n}\right) f^{(n)}(x)+\int f(x-t) d \mu(t) .
\end{aligned}
$$

It follows from Lemma 1 that the right side of this equation is differentiable, and that in fact

$$
f^{(N+1)}(x)=\sum_{n=0}^{N-1}\left(-a_{n}\right) f^{(n+1)}(x)+\int f^{\prime}(x-t) d \mu(t)
$$

Further,

$$
\begin{gathered}
\left|f^{(N+1)}(x)\right| \leqslant \sum_{n=1}^{N}\left|-a_{n-1}\right|\left|f^{(n)}(x)\right|+\int\left|f^{\prime}(x-t)\right| d|\mu|(t) \\
\Rightarrow\left|f^{(N+1)}(x)\right| \leqslant\left(\sum_{n=1}^{N}\left|a_{n-1}\right| M_{n}+M_{1} V\right) e^{\tau|x|}
\end{gathered}
$$

using Lemma 4, where the $M_{n}$ are as in Lemma 4. This process can be repeated indefinitely, proving the proposition. 
Remark. The Propositions 3.4 and 3.5 have shown that the equations $\Sigma_{n=0}^{N} a_{n} f^{(n)}(x)=\int f(x-t) d \mu(t)$ have solutions which are entire functions of exponential type when all but one of the $a_{n}$ are zero, $1 \leqslant n \leqslant N$, and are at least infinitely differentiable otherwise.

For entire functions of exponential type, equality in the above equation holds not only for real $x$ but for every complex $z$.

To see this, consider the equation above in the following two cases: (a) $f$ is entire of exponential type $\tau$ and bounded on the real axis, and $\mu$ is a complex measure; and (b) $f$ is entire of exponential type $\tau$, and $\int e^{\sigma|t|} d|\mu|(t)<\infty$ for some $\sigma>\tau$.

Note first that the convolution integrals have finite values for complex $z$. In case (a)

$$
\int|f(z-t)| d|\mu|(t) \leqslant M e^{\tau|y|} \cdot|\mu|(R)
$$

for $z=x+i y$ and $M=\max _{-\infty<x<+\infty}|f(x)|$ by [1, Theorem 6.2.4], and case (b) follows as in Theorem 2.1 .

If $f(z)$ is entire, certainly $\Sigma_{n=0}^{N} a_{n} f^{(n)}(z)$ is also. But for both cases, as in the proof of Theorem 2.1, then so is $\int f(z-t) d \mu(t)$. Thus if these two functions agree on the real axis, they agree everywhere and conversely.

\section{Characterizations for certain classes of entire functions of slow growth} on the real axis. We now wish to focus attention on entire functions whose growth on the real axis is slower than exponential. Let $B_{a}$ be the set of entire functions of exponential type $a$ which are bounded on the real line, and let $W_{a}$ be those functions in $B_{a}$ which are square-integrable on the real line.

In the following, the Fourier transform performs much the same role that the Laplace transform did in the previous analysis. Results here are more general in the sense that solutions of equations with polynomial differential operators can be more complicated than sums of exponential polynomials and can be, say, the space $B_{a}$ or $W_{a}$ itself.

This first theorem shows the importance of convolution, and hence these equations for $W_{a}$.

THEOREM 4.1. Let $T$ be a bounded linear operator on $W_{a}$. Then $\exists s_{T} \in$ $w_{a} \ni$

$$
\begin{aligned}
(T f)(x) & =\int f(x-t) s_{T}(t) d t \\
& =\frac{\pi}{a} \sum_{n=-\infty}^{+\infty} f\left(\frac{n \pi}{a}\right) s_{T}\left(x-\frac{n \pi}{a}\right) \quad \forall f \in W_{a} .
\end{aligned}
$$

The operator $T$ may have such a representation for various functions $s_{T}$ in $W_{a}$, but $s_{T}=T(\sin a t / \pi t)$ is an example. 
If further $s_{T} \in L^{1}(R)$, then $\|T\| \leqslant\left\|s_{T}\right\|_{L^{1}}$.

Note. This shows that every bounded linear operator on $W_{a}$ is represented by convolution, in either integral or series form with an element of $W_{a}$.

PROOF. Let $f, g \in W_{a}$ and $\lambda_{n}=n \pi / a$ for any integer $n$. Note that $\left\{(2 a)^{-1} e^{i \lambda} n^{x}\right\}_{n=-\infty}^{+\infty}$ is a complete orthonormal set in $L^{2}(-a, a)$.

We first show that

$$
\int f(t) \overline{g(t)} d t=\frac{\pi}{a} \sum_{n=-\infty}^{+\infty} f\left(\lambda_{n}\right) \overline{g\left(\lambda_{n}\right)}, \quad \text { for } \lambda_{n} \text { as above. }
$$

Since $f \in W_{a}$, the Paley-Wiener theorem shows that $f(z)=(1 / 2 \pi) \int e^{-i z t} F(t) d t$ for some $F(t) \in L^{2}(-a, a), F(t)=0$ for $|t|>a$. In particular,

Similarly,

$$
\begin{aligned}
& 2 \pi f\left(\lambda_{n}\right)=\int_{-a}^{a} e^{-i \lambda_{n} t} F(t) d t \\
& \quad \Rightarrow 2 \pi f\left(\lambda_{n}\right)=2 a \int_{-a}^{a} F(t)\left\{\frac{1}{2 a} e^{-i \lambda} n^{t}\right\} d t .
\end{aligned}
$$

$$
2 \pi g(z)=\int_{-a}^{a} e^{-i z t} G(t) d t, \quad G \in L^{2}(-a, a) .
$$

Thus on $L^{2}(-a, a)$ with inner product given by $(x, y)=2 a \int_{-a}^{a} x(t) \overline{y(t)} d t$, for $x, y \in L^{2}(-a, a)$, we have

$$
\begin{aligned}
2 a \int F(t) \overline{G(t)} d t & =\sum_{n=-\infty}^{+\infty}(2 \pi)^{2} f\left(\lambda_{n}\right) \overline{g\left(\lambda_{n}\right)} \\
\Rightarrow \int F(t) \overline{G(t)} & d t=\frac{2 \pi^{2}}{a} \sum_{n=-\infty}^{+\infty} f\left(\lambda_{n}\right) \overline{g\left(\lambda_{n}\right)} .
\end{aligned}
$$

But Parseval's theorem gives

$$
\begin{gathered}
\int F(t) \overline{G(t)} d t=\frac{1}{2 \pi} \int(2 \pi f(t))(2 \pi \overline{g(t)}) d t=2 \pi \int f(t) \overline{g(t)} d t \\
\Rightarrow \int f(t) \overline{g(t)} d t=\frac{\pi}{a} \sum_{n=-\infty}^{+\infty} f\left(\lambda_{n}\right) \overline{g\left(\lambda_{n}\right)}
\end{gathered}
$$

thus proving (16).

Define $\operatorname{sinca}(t)=\sin a t / \pi t \in W_{a}$ : It is known, for example [4], that the first part of (15) holds when $T$ is the identity operator. That is,

$$
f \in W_{a} \Rightarrow f(x)=\int f(x-t) \operatorname{sinca}(t) d t .
$$

Another proof of this fact is contained in Theorem 4.3.

Since $T$ is an operator on $W_{a}$, this implies that 


$$
\begin{aligned}
{[T f](x) } & =\int[T f](x-t) \operatorname{sinca}(t) d t \\
& =\int[T f](t) \operatorname{sinca}(x-t) d t=\frac{\pi}{a} \sum_{n=-\infty}^{+\infty}[T f]\left(\lambda_{n}\right) \operatorname{sinca}\left(x-\lambda_{n}\right)
\end{aligned}
$$

by (16).

Kramer [12] has shown that

$$
\sum_{n=-\infty}^{+\infty}[T f]\left(\lambda_{n}\right) \operatorname{sinca}\left(x-\lambda_{n}\right)=\sum_{n=-\infty}^{+\infty} f\left(\lambda_{n}\right)[T(\text { sinca })]\left(x-\lambda_{n}\right)
$$

holds for $a=\pi$ and $\lambda_{n}=n$, but his proof also works under these more general conditions. But using (16)

$$
\begin{aligned}
\frac{\pi}{a} \sum_{n=-\infty}^{+\infty} f\left(\lambda_{n}\right)[T(\text { sinca })]\left(x-\lambda_{n}\right) & =\int f(t)[T(\text { sinca })](x-t) d t \\
& =\int f(x-t)[T(\text { sinca })](t) d t
\end{aligned}
$$

Since $s_{T}=T[$ sinca $]$, we have

$$
[T f](x)=\int f(x-t) s_{T}(t) d t=\frac{\pi}{a} \sum_{n=-\infty}^{+\infty} f\left(\lambda_{n}\right) s_{T}\left(x-\lambda_{n}\right)
$$

so (15) is proved.

If $s_{T} \in L^{1}, f \in L^{2}$, then Young's inequality gives $\left\|f * s_{T}\right\|_{2} \leqslant\left\|s_{T}\right\|_{1}\|f\|_{2}$, i.e., $\|T f\|_{2} \leqslant\left\|s_{T}\right\|_{1}\|f\|_{2} \Rightarrow\|T\| \leqslant\left\|s_{T}\right\|_{1}$.

Proposition 4.2. Let $D^{n}$ be a differential operator of order $n$ on $W_{a^{\text {. }}}$. Then $\left\|D^{n}\right\|=a^{n}$.

Proof. By the Paley-Wiener theorem, an element of $W_{a}$ has the form $f(z)=\int_{-a}^{a} e^{i t z} g(t) d t$, for some $g \in L^{2}(-a, a)$. Thus

$$
D^{n} f(z)=\int_{-a}^{a}(i t)^{n} e^{i t z} g(t) d t .
$$

By Parseval's theorem

$$
\begin{aligned}
\left\|D^{n} f\right\|_{2}^{2} & =\int_{-a}^{a}|t|^{2 n}|g(t)|^{2} d t \leqslant a^{2 n} \int|g(t)|^{2} d t=a^{2 n}\|f\|_{2}^{2} \\
& \Rightarrow\left\|D^{n} f\right\|_{2} \leqslant a^{n}\|f\|_{2} \Rightarrow\left\|D^{n}\right\| \leqslant a^{n} .
\end{aligned}
$$

We now exhibit a sequence in $W_{a}$ which gives equality above: Let $f_{n}(z)=$ $\int_{-a}^{a} e^{i t z} g_{n}(t) d t$, where

$$
g_{n}(t)= \begin{cases}t^{n} & \text { if } a \cdot((n-1) / n) \leqslant t \leqslant a \\ 0 & \text { otherwise }\end{cases}
$$

Then 
and

$$
\begin{aligned}
\left\|D^{N} f_{n}\right\|_{2}^{2} & =\int_{-a}^{a}|t|^{2 N}\left|g_{n}(t)\right|^{2} d t=\int_{a((n-1) / n)}^{a} t^{2 N+2 n} d t \\
& =\frac{a^{2(N+n)+1}}{2(N+n)+1}\left\{1-\left(1-\frac{1}{n}\right)^{2(N+n)+1}\right\}
\end{aligned}
$$

$$
\left\|f_{n}\right\|_{2}^{2}=\int_{-a}^{a}\left|g_{n}(t)\right|^{2} d t=\int_{a((n-1) / n)}^{a} t^{2 n} d t=\frac{a^{2 n+1}}{2 n+1}\left\{1-\left(1-\frac{1}{n}\right)^{2 n+1}\right\} .
$$

Thus

$$
\begin{aligned}
& \lim _{n \rightarrow \infty} \frac{\left\|D^{N} f_{n}\right\|_{2}^{2}}{\left\|f_{n}\right\|_{2}^{2}}=\lim _{n \rightarrow \infty} \frac{a^{2(N+n)+1}}{2(N+n)+1} \frac{\left\{1-(1-1 / n)^{2(N+n)+1}\right\}}{\left\{1-(1-1 / n)^{2 n+1}\right\}} \frac{2 n+1}{a^{2 n+1}} \\
& \Rightarrow \lim _{n \rightarrow \infty}\left\{\frac{\left\|D^{N} f_{n}\right\|_{2}}{\left\|f_{n}\right\|_{2}}\right\}^{2}=a^{2 N} \lim _{n \rightarrow \infty} \frac{2 n+1}{2 n+2 N+1} \\
& \cdot \lim _{n \rightarrow \infty} \frac{\left\{1-(1-1 / n)^{2 N+2 n+1}\right\}}{\left\{1-(1-1 / n)^{2 n+1}\right\}} \\
& =a^{2 N} \cdot 1 \cdot \lim _{n \rightarrow \infty} \frac{(2 N+2 n+1)(1-1 / n)^{2(N+n)} n^{-2}}{(2 n+1)(1-1 / n)^{2 n}(n)^{-2}} \\
& \text { by L'Hospital } \\
& =a^{2 N} \cdot 1 \cdot \lim _{n \rightarrow \infty} \frac{2 N+2 n+1}{2 n+1}\left(1-\frac{1}{n}\right)^{2 N}=a^{2 N} \\
& \Rightarrow \lim _{n \rightarrow \infty} \frac{\left\|D^{N} f_{n}\right\|}{\left\|f_{n}\right\|}=a^{N}
\end{aligned}
$$

and the proposition is proved.

REMARK. We can combine the above proposition with the previous theorem to obtain the following:

$$
\text { If } f^{(n)}(x)=\int f(x-t) \varphi(t) d t \text { for } \varphi \in L^{1}(R) \text { and } n \geqslant 0 \text {, for every } f \in W_{a},
$$
then the exponential type of $f=a \leqslant\left\{\|\varphi\|_{1}\right\}^{1 / n}$.

For the above equation says that $D^{n} f=f * \varphi$, so the theorem applies to give $\left\|D^{n}\right\| \leqslant\|\varphi\|_{1}$. But then $a^{n}=\left\|D^{n}\right\| \leqslant\|\varphi\|_{1}$ by the proposition, which implies $a \leqslant\left\{\|\varphi\|_{1}\right\}^{1 / n}$.

Examples of this situation occur in the next section.

We note the analogy between this result and Theorem 3.2.

Given a square-integrable, $N$-times continuously differentiable function on the real line as $f(x)$, the following theorem states conditions on the measure such 
that equations of the form (17) characterize the class $w_{a}$. Moreover, if an equation of this form is satisfied for all functions in $W_{a}$, the measure must be of the particular form described below:

THEOREM 4.3. Let $f \in L^{2}(R) \cap C^{N}(R)$. Suppose $d \mu$ is a measure such that

$$
\hat{\mu}(t)=\left\{\begin{array}{l}
\sum_{n=0}^{N} a_{n}(i t)^{n} \quad \text { for }|t|<a \text { and } a_{n} \text { complex } \\
g(t) \quad \text { for }|t|>a, \text { where } g(t) \neq \sum_{n=0}^{N} a_{n}(i t)^{n} \\
\text { for any } t \text { such that }|t|>a .
\end{array}\right.
$$

Then

$$
\int f(x-t) d \mu(t)=\sum_{n=0}^{N} a_{n} f^{(n)}(x)
$$

if and only if $f \in W_{a}$.

Further, if (17) holds for every $f \in W_{a}$ but not for every $f \in W_{b}$ where $b>$ $a$, then $\hat{\mu}(t)$ has the form above.

Proof. First note that if $f \in W_{a}$, then all its derivatives are in $W_{a}$ and thus Fourier transforms of every derivative exist.

Suppose $f \in W_{a}$. Since $\hat{f}(t)=0$ for $|t|>a$, then (except possibly at $t=a$ )

$$
\begin{aligned}
\hat{f}(t)\left[\hat{\mu}(t)-\sum_{n=0}^{N} a_{n}(i t)^{n}\right] & =0 \\
\Rightarrow \hat{f}(t) \hat{\mu}(t) & =\sum_{n=0}^{N} a_{n}(i t)^{n} \hat{f}(t)=\sum_{n=0}^{N} a_{n} \hat{f}^{(n)}(t) .
\end{aligned}
$$

But $(f * d \mu)^{\wedge}=\hat{f} \hat{\mu}$, so we have

$$
(f * d \mu)^{\wedge}(t)=\left\{\sum_{n=0}^{N} a_{n} f^{(n)}(t)\right\}^{\wedge}
$$

Taking inverse transforms, we see that (17) holds.

Conversely, if (17) holds, we reverse the order of steps to obtain

$$
\hat{f}(t)\left[\hat{\mu}(t)-\sum_{n=0}^{N} a_{n}(i t)^{n}\right]=0 .
$$

By the assumptions on the measure $\mu$, this implies $\hat{f}(t)=0$ for $|t|>a$. Taking inverse transforms, we have

$$
f(x)=\int_{-a}^{a} e^{i x t}(2 \pi)^{-1} \hat{f}(t) d t
$$

so $f \in W_{a}$ by the Paley-Wiener theorem. 
Finally, if (17) holds for every $f \in W_{a}$, it certainly holds for the function $(\pi x)^{-1} \sin a x \in W_{a}$ whose Fourier transform equals 1 on the interval $(-a, a)$. The equality above shows that then $\hat{\mu}(t)=\sum_{n=0}^{N} a_{n}(i t)^{n}$ for $|t|<a$. If $\hat{\mu}(t)$ had this form for the interval $|t|<b$ where $b>a$, the previous argument would show that (17) holds for every $f \in W_{b}$. This would contradict the hypothesis, so that $\hat{\mu}(t)$ must have the form described.

ExAMPLES. I. The Fourier transform of the function $\sin a t / \pi t \in W_{a}$ is one on $[-a, a]$ and zero elsewhere. Thus for $d \mu(t)=\sin a t(\pi t)^{-1} d t$, the previous theorem shows that

$$
\int f(x-t) \frac{\sin a t}{\pi t} d t=f(x)=\frac{\pi}{a} \sum_{n=-\infty}^{+\infty} f\left(\frac{n \pi}{a}\right) \frac{\sin a(x-n \pi / a)}{\pi(x-n \pi / a)}
$$

where the latter equality follows from Theorem 4.1. This is the cardinal series, or Whittaker-Shannon interpolation formula. For functions in $L^{2}(R)$, it characterizes the class $W_{a}$.

The series does converge under slightly less restrictive conditions on the growth of the function, see Jagerman [10] for example.

II. We have

$$
\begin{aligned}
f^{\prime}(x) & =\int f(x-t) \frac{a t \cos a t-\sin a t}{\pi t^{2}} d t \\
& =\frac{\pi}{a} \sum_{n=-\infty}^{+\infty} f\left(\frac{n \pi}{a}\right) \frac{d}{d x} \frac{\sin a(x-n \pi / a)}{\pi(x-n \pi / a)}
\end{aligned}
$$

by Theorem 4.1, since differentiation is a bounded linear operator on $W_{a}$. For square-integrable functions, the fact that the Fourier transform of [at $\cos a t-\sin a t]\left(\pi t^{2}\right)^{-1}$ equals it for $|t|<a$ leads to a second proof of the first equality by use of Theorem 4.3. Further, this theorem shows that the above equation characterizes such functions as elements of $W_{a}$.

Boas $[1$, p. 221], has shown that

$$
f^{\prime}(z)=-a \sin (a z) \sum_{n=-\infty}^{+\infty}(-1)^{n} f\left(\frac{n \pi}{a}\right)(a z-n \pi)^{-2}+a f(z) \cot (a z)
$$

for $f \in W_{a}$. Using trigonometric identities such as $(-1)^{n} \sin a z=\sin (a z-n \pi)$, it follows that this equals

$$
\frac{1}{a} \sum_{n=-\infty}^{+\infty}\left[a\left(z-\frac{n \pi}{a}\right) \cos a\left(z-\frac{n \pi}{a}\right)-\sin a\left(z-\frac{n \pi}{a}\right)\right]\left(z-\frac{n \pi}{a}\right)^{-2} f\left(\frac{n \pi}{a}\right) .
$$

This is, of course, the series in (19).

III. Consider the operator $D^{2}+a^{2} I$ acting on $W_{a}$. One calculates that 


$$
\begin{aligned}
\left(D^{2}+a^{2} I\right) \frac{\sin a t}{\pi t} & =\frac{-a^{2} \sin a t}{\pi t}-\frac{2 a \cos a t}{\pi t^{2}}+\frac{2 \sin a t}{\pi t^{3}}+a^{2} \frac{\sin a t}{\pi t} \\
& =\frac{2}{\pi}\left\{\frac{\sin a t}{t^{3}}-\frac{a \cos a t}{t^{2}}\right\} .
\end{aligned}
$$

Thus for $f \in L^{2}(R)$, Theorems 4.1 and 4.3 show that

$$
\begin{aligned}
f^{\prime \prime}(x)+a^{2} f(x) & =\frac{2}{\pi} \int f(x-t)\left\{\frac{\sin a t}{t^{3}}-\frac{a \cos a t}{t^{2}}\right\} d t \\
& =\frac{2}{a} \sum_{n=-\infty}^{+\infty} f\left(\begin{array}{c}
n \pi \\
a
\end{array}\right)\left\{\frac{\sin (a x-n \pi)}{(x-n \pi / a)^{3}}-\frac{a \cos (a x-n \pi)}{(x-n \pi / a)^{2}}\right\}
\end{aligned}
$$

is characteristic of the class $W_{a}$.

The latter series appears to be analogous to one found by A. J. Macintyre $[14$, p. 4].

IV. Define a measure $d \mu(t)$ by

$$
d \mu(t)=\left\{\begin{array}{l}
(-1)^{k} a / k \text { if } t=k \pi / a, k= \pm 1, \pm 2, \ldots, \\
0 \text { otherwise. }
\end{array}\right.
$$

Then

$$
\begin{aligned}
\hat{\mu}(t) & =a \sum_{-\infty}^{-1} \frac{(-1)^{k}}{k} e^{-i k \pi t / a}+a \sum_{1}^{\infty} \frac{(-1)^{k}}{k} e^{-i k \pi t / a} \\
& =a \sum_{1}^{\infty} \frac{(-1)^{k+1}}{k}\left[e^{i k \pi t / a}-e^{-i k \pi t / a}\right] \\
& =i a \cdot 2 \sum_{1}^{\infty} \frac{(-1)^{k+1}}{k} \sin k \pi t / a \\
& =i a(t / a) \text { for }|t|<a .
\end{aligned}
$$

So $\hat{\mu}(t)=i t$ for $|t|<a$, and for $|t|>a$ the graph repeats.

Thus this measure is of the form prescribed in Theorem 4.3, and

$$
\int f(x-t) d \mu(t)=a \sum_{k=-\infty ; k \neq 0}^{+\infty} \frac{(-1)^{k}}{k} f\left(x-\frac{k \pi}{a}\right)
$$

is a series which converges if $f \in L^{2}(R)$.

For example, to show the convergence of the series

$$
\sum_{k=1}^{\infty} \frac{\left|(-1)^{k}\right|}{k}\left|f\left(x-\frac{k \pi}{a}\right)\right|,
$$

we may consider the integral

$$
\int_{1}^{\infty} \frac{|f(x-\pi t / a)|}{t} d t
$$


By Hölder's inequality,

$$
\int_{1}^{\infty} \frac{|f(x-\pi t / a)|}{t} d t \leqslant\left\{\int_{1}^{\infty}\left|f\left(x-\frac{\pi t}{a}\right)\right|^{2} d t\right\}^{1 / 2}\left\{\int_{1}^{\infty} \frac{d t}{t^{2}}\right\}^{1 / 2}<\infty .
$$

Similarly, one can show that the part of the series when $k$ is negative is convergent. Thus

$$
f^{\prime}(x)=a \sum_{k=-\infty ; k \neq 0}^{+\infty} \frac{(-1)^{k}}{k} f\left(x-\frac{k \pi}{a}\right)
$$

for $f \in L^{2}(R) \cap C^{1}(R)$ is characteristic of $f \in W_{a}$.

Kramer [12] has shown that this particular series holds for $f \in W_{\pi}$ in the case that $x$ is an integer.

V. Define a measure $d \mu(t)$ by

$$
d \mu(t)=\left\{\begin{array}{l}
-a^{2} / 3 \quad \text { if } t=0 \\
\frac{2 a^{2}}{\pi^{2}} \frac{(-1)^{k+1}}{k^{2}} \text { if } t=\frac{k \pi}{a}, k= \pm 1, \pm 2, \ldots, \\
0 \text { otherwise. }
\end{array}\right.
$$

Then

$$
\begin{aligned}
\mu(t) & =-\frac{a^{2}}{3}+\frac{2 a^{2}}{\pi^{2}} \sum_{k=-\infty ; k \neq 0}^{+\infty} \frac{(-1)^{k+1}}{k^{2}} e^{-i k \pi t / a} \\
& =-\frac{a^{2}}{3}-\frac{2 a^{2}}{\pi^{2}} \sum_{k=-\infty ; k \neq 0}^{+\infty} \frac{(-1)^{k}}{k^{2}}\left\{\cos \left(\frac{k \pi t}{a}\right)-i \sin \left(\frac{k \pi t}{a}\right)\right\} \\
& \Rightarrow-\hat{\mu}(t)=\frac{a^{2}}{3}+\frac{2 a^{2}}{\pi^{2}} 2 \sum_{k=1}^{\infty} \frac{(-1)^{k}}{k^{2}} \cos \left(\frac{k \pi t}{a}\right)=t^{2} \quad \text { for }|t| \leqslant a,
\end{aligned}
$$

i.e., $\hat{\mu}(t)=(i t)^{2}$ for $|t| \leqslant a$, and the series repeats for $|t|>a$. Thus

$$
f^{\prime \prime}(x)=-\frac{a^{2} f(x)}{3}+\frac{2 a^{2}}{\pi^{2}} \sum_{k=-\infty ; k \neq 0}^{+\infty} \frac{(-1)^{k}}{k^{2}} f\left(x-\frac{k \pi}{a}\right)
$$

for $f \in L^{2}(R) \cap C^{2}(R)$ is characteristic of $f \in W_{a}$.

But note that the series converges under the weaker assumption that $f$ is only bounded. Since

$$
|\mu|(R)=\frac{a^{2}}{3}+\frac{2 a^{2}}{\pi^{2}} \sum_{k=-\infty ; k \neq 0}^{+\infty} \frac{1}{k^{2}}=\frac{a^{2}}{3}+\frac{4 a^{2}}{\pi^{2}}\left(\frac{\pi^{2}}{6}\right)=\frac{a^{2}}{3}+\frac{2 a^{2}}{3}=a^{2},
$$

Theorem 3.1 shows that if $f(x)$ is a bounded function which satisfies (22), then $f$ is entire of exponential type $a$, or $f \in B_{a}$ as defined at the beginning of $\S 4$.

We would like to show that every $f \in B_{a}$ satisfies (22). If this occurs, then 
for bounded functions, this equation will characterize $B_{a}$. That fact is a consequence of the following more general theorem.

THEOREM 4.4. Let $d \mu$ be a measure such that

$$
\hat{\mu}(t)=\left\{\begin{array}{l}
\sum_{n=0}^{N} a_{n}(i t)^{n} \quad \text { for }|t| \leqslant a, \\
g(t) \quad \text { for }|t|>a, \text { where } g(t) \neq \sum_{n=0}^{N} a_{n}(i t)^{n} \\
\text { for any } t \text { such that }|t|>a .
\end{array}\right.
$$

Further suppose that $\int|t|^{J} d|\mu|(t)<\infty$ for some $J \geqslant 0$. Let $f \in C^{N}(R)$ be such that $f(x)=O\left(|x|^{J}\right)$ as $|x| \rightarrow \infty$. Then

$$
\int f(x-t) d \mu(t)=\sum_{n=0}^{N} a_{n} f^{(n)}(x)
$$

if and only if $f$ is entire of exponential type a. Further, if (23) does hold for this class of functions, then $\hat{\mu}(t)=\Sigma_{n=0}^{N} a_{n}(i t)^{n}$ for $|t| \leqslant a$.

Proof. First note that the assumptions on the growth of $f(x)$ and on the measure assure the existence of the convolution integral for all $x$. We may consider $f$ as a tempered distribution, that is an element of $S^{\prime}$ where $S^{\prime}$ is the set of continuous linear functionals on the space of rapidly decreasing functions on the real line.

Suppose first that $f$ is entire of exponential type $a$. As a functional in $S^{\prime}$, it has a Fourier transform $\hat{f}$ in $S^{\prime}$ whose support is in the interval $[-a, a]$. The convolution $f * d \mu$ is also a distribution, and $(f * d \mu)^{\wedge}=\hat{\mu} \hat{f}$. By the hypothesis on the measure,

$$
\hat{\mu} \hat{f}=\sum_{n=0}^{N} a_{n}(i t)^{n} \cdot \hat{f}=\left(\sum_{n=0}^{N} a_{n} f^{(n)}\right) \hat{~}
$$

so

$$
(f * d \mu)^{\wedge}=\left(\sum_{n=0}^{N} a_{n} f^{(n)}\right) \hat{.}
$$

Taking inverse transforms, this gives (23) as distributions and thus as functions.

Conversely, suppose that (23) holds. We have that

$$
(f * d \mu)^{\wedge}=\left(\sum_{n=0}^{N} a_{n} f^{(n)}\right) \hat{=}=\sum_{n=0}^{N} a_{n}(i t)^{n} \hat{f} .
$$

So

$$
\hat{\mu} \hat{f}=\sum_{n=0}^{N} a_{n}(i t)^{n} \hat{f} \text { and }\left(\hat{\mu}-\sum_{n=0}^{N} a_{n}(i t)^{n}\right) \hat{f}=0
$$


By the hypothesis on $\hat{\mu}$, we have $\hat{f}=0$ outside the interval $[-a, a]$. Thus the support of $\hat{f}$ is in the interval $[-a, a]$. Hence by $[18$, p. 183], the function $g(z)$ $=\hat{f}\left(e^{-i t z}\right)$ is entire of exponential type $a$ and the restriction of $g(z)$ to the real axis is the Fourier transform of $\hat{f}$. Of course, the Fourier transform of $\hat{f}$ is nearly $f$; the relation between them as distributions is given by $(\hat{f})^{\wedge}[\varphi(x)]=f(\varphi(-x))$ where $\varphi$ is an element of the space of rapidly decreasing functions on the real line. Thus $g(-z)$ is an entire function of exponential type $a$ which is equal to the original function $f(x)$ on the real axis. Hence $f(x)$ extends to an entire function of exponential type $a$, as claimed.

Finally, if (23) does hold for the set of entire functions of exponential type $a$ such that $f(x)=O\left(|x|^{J}\right)$ as $|x| \rightarrow \infty$ for any fixed $J \geqslant 0$, then it holds for the functions $e^{i k z}$ for $|k| \leqslant a$. The characterization of the measure follows.

ExAmples. I(a). The function $\operatorname{cosc}(t)=[\cos (a t)-\cos (a+\epsilon) t] / \epsilon \pi t^{2}$ for $\epsilon>0$ given has the Fourier transform

$$
[\operatorname{cosc}(t)]^{\wedge}=\left\{\begin{array}{l}
1 \quad \text { if }|t| \leqslant a \\
\epsilon^{-1}(t+a+\epsilon) \quad \text { if }-a-\epsilon \leqslant t \leqslant-a \\
\epsilon^{-1}(-t+a+\epsilon) \text { if } a \leqslant t \leqslant a+\epsilon \\
0 \text { otherwise, }
\end{array}\right.
$$

and $\operatorname{cosc}(t)$ is in $L^{1}(R)$. If $f$ is a bounded function on the real line, the previous theorem shows that

$$
\int f(x-t) \operatorname{cosc}(t) d t=f(x)
$$

is characteristic of $B_{a}$.

Note that (24) holds for $f \in W_{a}$, so the remark following Proposition 4.2 shows that $1 \leqslant\|\operatorname{cosc}(t)\|_{L 1}$.

I(b). Since $\operatorname{cosc}(t)$ is an $L^{1}(R)$ function which is also entire of exponential type, Theorem 11.3.3 of [1] shows that each derivative of $\operatorname{cosc}(t)$ is also in $L^{1}(R)$. The relations

$$
\left[d^{n} \operatorname{cosc}(t) / d t^{n}\right]^{\wedge}=(i t)^{n}[\operatorname{cosc}(t)]^{\wedge}
$$

hold for $n \geqslant 1$, so for any bounded function on the real line, the equation

$$
\int f(x-t) \frac{d^{n}}{d t^{n}}\{\operatorname{cosc}(t)\} d t=f^{(n)}(x)
$$

is characteristic of $B_{a}$.

Again the note following Proposition 4.2, or Theorem 3.2, shows that

$$
a \leqslant\left\|d^{n}\{\operatorname{cosc}(t)\} / d t^{n}\right\|_{L_{1}^{1 / n}}^{1} \text { for } n \geqslant 1 \text {. }
$$

We proceed to develop several different examples for the case that the growth 
of the function $f(x)$ in Theorem 4.4 for real $x$ may be any power of $|x|$ as $|x|$ $\rightarrow \infty$.

II(a). Suppose $f \in C^{n}(R)$ and $f(x)=O\left(|x|^{J-1}\right)$ for $J \geqslant 1$ as $|x| \rightarrow \infty$. Then

$$
\begin{aligned}
& f^{(n)}(x)=\int f(x-t)\left[\frac{\sin (\delta t / J)}{\delta t / J}\right]^{J} \frac{d^{n}}{d t^{n}}\left\{\frac{\sin (a+\delta) t}{\pi t}\right\} d t \\
& \\
& n \geqslant 0, \delta>0,
\end{aligned}
$$

if and only if $f$ is entire of exponential type $a$.

We first consider the case $n=0$. Let $g(t)=[h(t)]^{J} h_{1}(t)$ where $h(t)=$ $\sin (\delta t / J)[\delta t / J]^{-1}$ and $h_{1}(t)=\sin \left(a_{1} t\right)[\pi t]^{-1}$ where $a_{1}=a+\delta$. To show (26), we need to compute $\hat{g}(t)$, but under our system of Fourier transforms we have $\left(h h_{1}\right)^{\wedge}=(2 \pi)^{-1}\left(\hat{h} * \hat{h}_{1}\right)$. We note that $h(t)=(\pi J / \delta)(\sin (\delta t / J) / \pi t)$ has transform

$$
\hat{h}(t)=\left\{\begin{array}{l}
\pi J / \delta \text { if }|t| \leqslant \delta / J \\
0 \text { otherwise }
\end{array}\right.
$$

and

$$
\hat{h}_{1}(t)= \begin{cases}1 & \text { if }|t| \leqslant a_{1} \\ 0 & \text { otherwise }\end{cases}
$$

Now

$$
\begin{aligned}
\left(h h_{1}\right)^{\wedge} & =(2 \pi)^{-1} \int \hat{h}(x-t) \hat{h}_{1}(t) d t=(2 \pi)^{-1} \int_{-a_{1}}^{a_{1}} \hat{h}(x-t) d t \\
& =(2 \pi)^{-1} \int_{x-a_{1}}^{x+a_{1}} \hat{h}(t) d t .
\end{aligned}
$$

So if $|x| \leqslant a_{1}-\delta / J$,

$$
\left(h h_{1}\right)^{\wedge}=(2 \pi)^{-1} \int_{-\delta / J}^{\delta / J} \hat{h}(t) d t=(2 \pi)^{-1} \frac{\pi J}{\delta} \frac{2 \delta}{J}=1 .
$$

If $a_{1}-\delta / J \leqslant x \leqslant a_{1}+\delta / J$

$$
(2 \pi)^{-1} \int_{x-a_{1}}^{x+a_{1}} \hat{h}(t) d t=\left(a_{1}+\delta / J\right)-x
$$

So pictorially, $\left(h h_{1}\right)^{\wedge}$ is

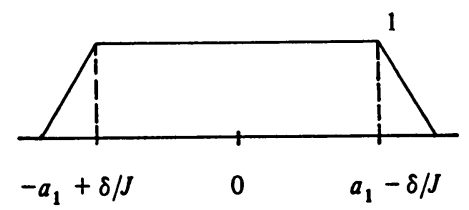

Continuing, $\left(h_{1} h^{2}\right)^{\wedge}=(2 \pi)^{-1}\left(\left(h_{1} h\right)^{\wedge} * \hat{h}\right)=(2 \pi)^{-2}\left(\left(\hat{h}_{1} * \hat{h}\right) * \hat{h}\right)$, so in general 


$$
\left(h_{1} h^{J}\right)^{\wedge}=(2 \pi)^{-J}\left(\left(\hat{h}_{1} * \hat{h}\right) * \hat{h} * \cdots * \hat{h}\right)
$$

the convolution repeated $J$ times. Graphically, we have

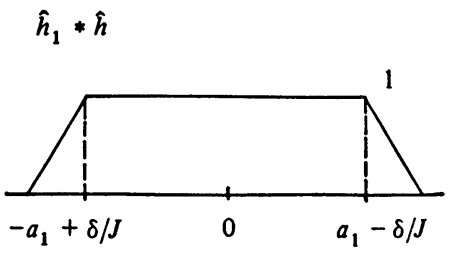

$J$ th convolution

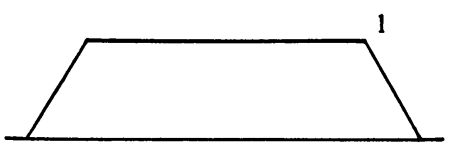

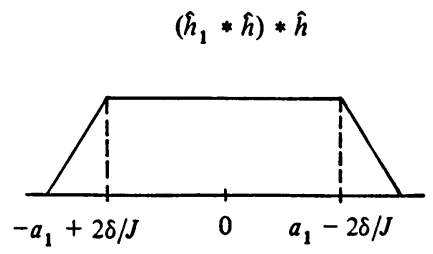

$* \hat{h}$
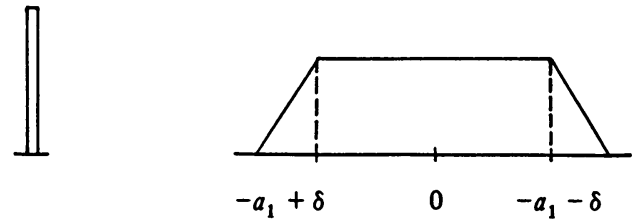

or

$$
\hat{g}=\left(h_{1} h^{J}\right)^{\wedge}=\left\{\begin{array}{l}
1 \quad \text { if }|t| \leqslant a_{1}-\delta=a, \\
r(t) \text { where }|r(t)|<1 \text { for }|t|>a
\end{array}\right.
$$

and the case $n=0$ follows from the theorem.

For derivatives of order $n>0$, we know that the Fourier transform of the $n$th derivative of $\sin \left(a_{1} t\right)[\pi t]^{-1}$ is $(i t)^{n}$ for $|t| \leqslant a_{1}$ and zero outside this interval. The same process as above shows that the integrand product defined in (26) has a transform equal to $(i t)^{n}$ on $|t| \leqslant a$ which is not (it) ${ }^{n}$ for any $t$ such that $|t|>a$. Thus (26) also follows for any $n>0$.

Note. D. Jagerman [10] has used a different method to find the following series:

$$
f(t)=\sum_{n=-\infty}^{+\infty} f(n h)\left\{\frac{\sin \delta a^{\prime}(t-n h) / m}{\delta a^{\prime}(t-n h) / m}\right\}^{m} \frac{\sin a^{\prime}(t-n h)}{a^{\prime}(t-n h)}
$$

where $f \in B_{a}, a^{\prime}=a /(1-\delta)$ for $\delta>0, h=\pi / a^{\prime}$, and $m>0$. Jagerman shows that every $f \in B_{a}$ satisfies this series.

Since

$$
a^{\prime}-m\left(\delta a^{\prime} / m\right)=\frac{a}{1-\delta}-\frac{\delta a}{1-\delta}=\frac{a(1-\delta)}{1-\delta}=a,
$$

the method used above in this example to derive (26) can be used again to derive the similar equation 


$$
f^{(n)}(x)=\int f(x-t)\left\{\frac{\sin \left(\delta a^{\prime} t / m\right)}{\delta a^{\prime} t / m}\right\}^{m} \frac{d^{n}}{d t^{n}}\left\{\frac{\sin a^{\prime} t}{\pi t}\right\} d t
$$

with the hypothesis and conclusions of (26) where $a^{\prime}$ and $m$ are as above. An application of (16) to the case when $f \in W_{a}$ (so certainly $f \in W_{a^{\prime}}$ ) and $n=0$ shows that this integral equals Jagerman's series. For functions $f \in B_{a}$ or of more rapid growth on the real line, it would appear to require a different technique to show the equality of the integral and the series.

EXAMPLE II(b). Now suppose $f \in C^{n}(R)$ and $f(x)=O\left(|x|^{J}\right)$ for $J \geqslant 0$ as $|x| \rightarrow \infty$. Then

$$
f^{(n)}(x)=\int f(x-t)\left\{\frac{\sin (\delta t / J)}{\delta t / J}\right\}^{J} \frac{d^{n}}{d t^{n}}\left\{\operatorname{cosca}_{1}(t)\right\} d t
$$

where $n \geqslant 0, \delta>0$ and

$$
\operatorname{cosca}_{1}(t)=[\cos (a+\delta) t-\cos (a+\epsilon+\delta) t] / \epsilon \pi t^{2}
$$

for some $\epsilon>0$, if and only if $f$ is entire of exponential type $a$.

The case $J=0$ (where we then assume $\delta=0$ ) is Example I. For $J>0$, the proof of (27) is a result of combining the proof of Example I, the procedure of Example II(a), and the previous theorem.

EXAMPLE III. This final example also concerns the case when the function has polynomial growth of any degree on the real line.

Let $A$ be the compact set $[-a, a]$, and $B$ the open set $(-a-\epsilon, a+\epsilon)$ for $\epsilon>0$ small. Friedman $[5$, p. 45], has shown that there exists an infinitely differentiable function $\beta(x)$ such that (i) $\beta(x)=1$ if $x$ is in $A$, (ii) $\beta(x)=0$ if $|x| \geqslant$ $a+\epsilon$, and (iii) $0 \leqslant \beta(x) \leqslant 1$ for all $x$. We pick $\epsilon$ small enough so that $|\beta(x)|<1$ if $|x|>a$.

A formula for such a function is given by $f_{1} * f_{2}$, where

$$
f_{1}(x)= \begin{cases}\frac{2 k}{\epsilon} \exp \frac{-1}{1-(2 x / \epsilon)^{2}} & \text { if }|x| \leqslant \frac{\epsilon}{2}, \\ 0 & \text { otherwise. }\end{cases}
$$

and

$$
f_{2}(x)= \begin{cases}1 & \text { if }|x| \leqslant a+\epsilon / 2 \\ 0 & \text { otherwise }\end{cases}
$$

Define

$$
K a(x)=\int e^{i x t} \beta(t) d t=\int_{B} e^{i x t} \beta(t) d t ;
$$

i.e., $K a$ is the inverse transform of $\beta$. Further, it is an entire function of exponential type which is in $L^{2}(R)$. Also, $K a$ has Fourier transform $\beta$. 
An integration by parts shows that

$$
K a(x)=\int_{B} e^{i x t} \beta(t) d t=0-(i x)^{-1} \int_{B} e^{i x t} \beta^{\prime}(t) d t .
$$

This can be repeated any number of times, so that

$$
K a(x)=(-1)^{n}(i x)^{-n} \int_{B} e^{i x t} \beta^{(n)}(t) d t \text { for } n \geqslant 0 .
$$

Thus $|K a(x)| \leqslant|x|^{-n} \int_{B}\left|\beta^{(n)}(t)\right| d t$ for arbitrary $n$, so that

$$
\int|t|^{J}|K a(t)| d t<\infty \quad \text { for } J \geqslant 0
$$

Since $K a^{(n)}(x)=\int_{B}(i t)^{n} e^{i x t} \beta(t) d t$, a similar argument shows that $\int|t|^{J}\left|K a^{(n)}(t)\right| d t<\infty$ for any $n \geqslant 0$ and $J \geqslant 0$. Using the theorem, we conclude that

$$
f^{(n)}(x)=\int f(x-t) K a^{(n)}(t) d t, \quad n \geqslant 0,
$$

characterizes entire functions of exponential type which have polynomial growth on the real line.

At this point, we give a further condition which characterizes the measures in the case that an equation of the form (5), i.e., equations as in the following theorem, always holds. We first consider the case when the measure decays exponentially, as in $\S 2$. We then present an example to suggest that similar results may hold in a more generalized sense for measures of slower decay.

THEOREM 4.5. Let $d \mu$ be such that $\int e^{\sigma|t|} d|\mu|(t)<\infty$ for some $\sigma>0$. Then

$$
(f * d \mu)(x)=\sum_{n=0}^{N} a_{n} f^{(n)}(x)
$$

for every entire function of exponential type $a(a<\sigma)$ if and only if

$$
\int(-t)^{k} d \mu(t)=\left\{\begin{array}{l}
k ! a_{k} \quad \text { if } k \geqslant 0, k \leqslant N, \\
0 \quad \text { if } k>N .
\end{array}\right.
$$

PROOF. If the equation holds for every entire function of exponential type $a$, then it holds if $f(x)=x^{k}$ for $k \geqslant 0$. But then

$$
\int(x-t)^{k} d \mu(t)=\sum_{n=0}^{k} a_{n} \frac{k !}{(k-n) !} x^{k-n},
$$

where $a_{n}=0$ if $n>N$. Setting $x=0$ gives $\int(-t)^{k} d \mu(t)=k ! a_{k}$.

Conversely, suppose that the moment condition holds for $k \geqslant 0$. If $P(x)$ is any polynomial of degree $M$, say, then $P(x-t)$ for $x$ fixed is of the form 


$$
P(x-t)=\sum_{n=0}^{M} \frac{P^{(n)}(x)}{n !}(-t)^{n}
$$

Thus

$$
\begin{aligned}
\int P(x-t) d \mu(t) & =\int \sum_{n=0}^{M} \frac{P^{(n)}(x)}{n !}(-t)^{n} d \mu(t) \\
& =\sum_{n=0}^{M} \frac{P^{(n)}(x)}{n !} \int(-t)^{n} d \mu(t)=\sum_{n=0}^{N} a_{n} P^{(n)}(x),
\end{aligned}
$$

so that the equation holds for all polynomials.

Claim.

$$
\int\left\{f(x-t)-\sum_{n=0}^{N} \frac{f^{(n)}(x)}{n !}(-t)^{n}\right\} d \mu(t)=0
$$

for every entire function of exponential type $a<\sigma$. To show this, let $X=$ $\left\{g \in L^{1}(d|\mu|): g(t)\right.$ is entire , and $M=\{p: p(t)$ is a polynomial $\}$. We have that $M$ is a subspace of the Banach space $X$, and the hypotheses assure us that every entire function of exponential type $a$ is in $X$.

Fix $x$, and define a linear functional $\Phi$ on $X$ by

$$
\Phi[g]=\int\left\{g(x-t)-\sum_{n=0}^{N} \frac{g^{(n)}(x)}{n !}(-t)^{n}\right\} d \mu(t) .
$$

$\Phi$ is a continuous linear functional on $X$. This functional has the property that $\Phi[p]=0 \forall p \in M$. By the Hahn-Banach theorem, $\Phi[g]=0$ for every $g \in \bar{M}$, the closure of $M$ in $X$.

If $f$ is any entire function of exponential type $a$, and $x$ is fixed, then $f_{x}(t)$ $=f(x-t)$ is also of this class, and thus in $X$. It is clear that

$$
f_{x}(t)=\sum_{k=0}^{\infty} \frac{f^{(k)}(x)}{k !}(-t)^{k}
$$

is the pointwise limit of polynomials

$$
p_{n}(t)=\sum_{k=0}^{n} \frac{f^{(k)}(x)}{k !}(-t)^{k}
$$

We claim that $f_{x}$ is also the norm limit of the polynomials $p_{n}$.

Since $f_{x}$ is of exponential type less than $\sigma,\left|f^{(k)}(x)\right| \leqslant \sigma^{k}$ for fixed $x$ and large $k$. Thus

$$
\left|f_{x}(t)\right| \leqslant M \sum_{k=0}^{\infty} \frac{\sigma^{k}|t|^{k}}{k !}
$$


for some constant $M$, i.e., $\left|f_{x}(t)\right| \leqslant M e^{\sigma|t|}$. Moreover, $\left|p_{n}(t)-f_{x}(t)\right| \leqslant N e^{\sigma|t|}$ for large $n$ and constant $N$. Thus

$$
\left\|p_{n}-f_{x}\right\|_{1}=\int\left|p_{n}(t)-f_{x}(t)\right| d|\mu|(t) \rightarrow 0
$$

by the dominated convergence theorem. Hence $f_{x}$ is the norm limit of polynomials $p_{n}$, so $f_{x} \in \bar{M}$, and the claim follows.

By the claim,

$$
\begin{aligned}
\int f(x-t) d \mu(t) & =\int \sum_{n=0}^{N} \frac{f^{(n)}(x)}{n !}(-t)^{n} d \mu(t) \\
& =\sum_{n=0}^{N} \frac{f^{(n)}(x)}{n !} \int(-t)^{n} d \mu(t)=\sum_{n=0}^{N} a_{n} f^{(n)}(x)
\end{aligned}
$$

as claimed.

Notes. (1) Example 3 following Theorem 3.1 concerned a measure defined by

$$
d \mu(t)=\left\{\begin{array}{l}
\frac{4 \tau(-1)^{n}}{\pi^{2}(2 k+1)^{2}} \text { if } t=-\left(\frac{2 k+1}{2 \tau}\right) \pi, \quad k=0, \pm 1, \ldots, \\
0 \text { otherwise. }
\end{array}\right.
$$

Recall that for this measure and $f(x)$ in $B_{\tau}, \int f(x-t) d \mu(t)=f^{\prime}(x)$. The moments of this measure do not all exist in the ordinary sense. But

$$
\int d \mu(t)=\frac{4 \tau}{\pi^{2}} \sum_{k=-\infty}^{+\infty} \frac{(-1)^{k}}{(2 k+1)^{2}}=0, \quad \int(-t) d \mu(t)=\frac{2}{\pi} \sum_{k=-\infty}^{+\infty} \frac{(-1)^{k}}{2 k+1}=1
$$

and for $n \geqslant 2$,

$$
\int(-t)^{n} d \mu(t)=\frac{4 \tau}{\pi^{2}}\left(\frac{\pi}{2 \tau}\right)^{n} \sum_{k=-\infty}^{+\infty}(-1)^{n}(2 k+1)^{n-2}
$$

is Abel summable with sum zero.

The analogy between the example and Theorem 4.5 is that if the moments of the measure did exist in the ordinary sense, the equation $f * d \mu=f^{\prime}$ would lead Theorem 4.5 to assign them the exact values as above. Thus a condition similar to that of Theorem 4.5 may hold in a more generalized sense for measures whose moments are not all finite.

(2) Combining Theorem 4.5 with Theorem 2.1, we see that for measures $d \mu$ such that $\int e^{\sigma|t|} d|\mu|(t)<\infty$ for some $\sigma>0$,

in a disc if and only if

$$
\int e^{-z t} d \mu(t)=\sum_{n=0}^{N} a_{n} z^{n}
$$




$$
\int(-t)^{k} d \mu(t)=\left\{\begin{array}{l}
k ! a_{k} \text { for } 0 \leqslant k \leqslant N \\
0 \text { otherwise. }
\end{array}\right.
$$

5. An application. Finally, a classical theorem states that if $f$ is an entire function which satisfies both $f(x+\tau)=f(x)$ and $f\left(x+\tau_{1}\right)=f(x)$ where $\tau / \tau_{1}$ is irrational, then $f$ is constant. Gel'fond [6] has considered a generalization of this by considering entire functions which satisfy two infinite linear differential equations. But if $d \mu$ is the measure which is point mass at $-\tau$, then

$$
\int f(x-t) d \mu(t)=f(x+\tau),
$$

so that a periodic function satisfies a particular convolution equation. The following theorem is a generalization of the classical theorem in terms of convolution equations.

We will consider the following cases, where $\mu$ represents the measure in each case:

Case (i). $f \in L^{2}(R)$ and $\hat{\mu}(t)$ exists.

Case (ii). $f(x)=O\left(|x|^{J}\right)$ for $J \geqslant 0$ as $|x| \rightarrow \infty$, and $\hat{\mu}(t)$ exists.

Case (iii). $f(x)$ has arbitrary growth, but $\left|\int e^{-z t} d \mu(t)\right|<\infty$ for $|\operatorname{Re} z| \leqslant b$, $b>a$.

THEOREM 5.1. Let $f(z)$ be entire of exponential type a. Suppose $f(z)$ satisfies both

$$
\sum_{n=0}^{N} a_{n} f^{(n)}(x)=\int f(x-t) d \mu(t) \text { and } \sum_{k=0}^{K} b_{k} f^{(k)}(x)=\int f(x-t) d \mu_{1}(t)
$$

where for Cases (i) and (ii), the set of common zeros of the functions

$$
\hat{\mu}(t)-\sum_{n=0}^{N} a_{n}(i t)^{n} \quad \text { and } \quad \hat{\mu}_{1}(t)-\sum_{k=0}^{K} b_{k}(i t)^{k} \quad \text { for }|t| \leqslant a
$$

is at most the origin (where roots of multiplicity $m$ and $m_{1}$ occur) and for Case (iii) the set of common zeros of the complex functions

$$
\int e^{-z t} d \mu(t)-\sum_{n=0}^{N} a_{n} z^{n} \text { and } \int e^{-z t} d \mu_{1}(t)-\sum_{k=0}^{K} b_{k} z^{k}
$$

is at most the origin (where roots of multiplicity $m$ and $m_{1}$ occur). Then, Case (i). $f$ is identically zero, Cases (ii) and (iii). $f$ is a polynomial of degree $\min \left(m-1, m_{1}-1\right)$.

Proof. Case (i). As before, the equation

$$
\hat{f}(t)\left(\hat{\mu}(t)-\sum_{n=0}^{N} a_{n}(i t)^{n}\right)=0
$$

holds. Similarly, 


$$
\hat{f}(t)\left(\hat{\mu}_{1}(t)-\sum_{k=0}^{K} b_{k}(i t)^{k}\right)=0 .
$$

Since $\hat{f}(t)=0$ for $|t|>a$, the hypotheses show $\hat{f}(t)=0$ except possibly at the origin. Thus $f=0$.

Case (ii). The hypothesis $f(x)=O\left(|x|^{J}\right)$ as $|x| \rightarrow \infty$ enables $f$ to be considered as a tempered distribution. As in a previous proof, we obtain

$$
\left(\hat{\mu}(t)-\sum_{n=0}^{N} a_{n}(i t)^{n}\right) \hat{f}=0 \quad \text { and } \quad\left(\hat{\mu}_{1}(t)-\sum_{k=0}^{K} b_{k}(i t)^{k}\right) \hat{f}=0 .
$$

So, by the fact that the support of $\hat{f}$ is the interval $[-a, a]$ and the hypotheses on the measures, $\hat{f}$ has support only at the origin. Rudin $[18$, p. 178] states that "a distribution is the Fourier transform of a polynomial if and only if its support is the origin (or the empty set)". Since the support of $\hat{f}$ is the origin, this makes $\hat{f}$ the Fourier transform of a polynomial. In fact, if $\hat{f}$ has order $N_{1}$, then Theorem 6.25 of [18] gives $\hat{f}=\Sigma_{k=0}^{N} c_{k}(i)^{-k} D^{k} \delta$, where $\delta$ is the impulse distribution.

Let $g(t)=\hat{\mu}(t)-\Sigma_{n=0}^{N} a_{n}(i t)^{n}$, and we have assumed that the multiplicity of the root of $g(t)$ at 0 is $m$. The above analysis says that $g(t) \hat{f}$ is the zero distribution, i.e.,

$$
\left\{g(t) \cdot \sum_{k=0}^{N_{1}} c_{k}(i)^{-k} D^{k} \delta\right\}(\varphi)=0 \quad \text { for } \varphi \in S,
$$

i.e., for every test function $\varphi$. But this means that $\Sigma_{k=0}^{N_{1}} c_{k}(i)^{-k}\left(D^{k}\{g \varphi\}\right)(0)=0$ for every $\varphi \in S$. But $g^{(n)}(0)$ is zero for $n \leqslant m$ only, so $\left\{D^{k}(g \varphi)\right\}(0)$ could not be zero for every $\varphi \in S$ and $k \leqslant N_{1}$. Hence for the above equation to hold, it must be true that $c_{k}=0$ if $k>m$. Thus $\hat{f}=\Sigma_{k=0}^{m} c_{k}(i)^{-k} D^{k} \delta$, and taking inverse transforms gives $f(x)=\sum_{k=0}^{m} c_{k} x^{k-1}$. Since this procedure could also be done with $g(t)$ defined in terms of $\hat{\mu}_{1}(t)$, etc., we have that $f$ is a polynomial of degree at most $\min \left(m-1, m_{1}-1\right)$.

Case (iii). Since $f(z)$ is entire, it is of the form $\int_{C} e^{z \omega} \varphi(\omega) d \omega$, where $\varphi$ is the Borel transform of $f$, and $C$ is a contour enclosing a region which contains the conjugate indicator diagram of $f$.

Following the proof of Proposition 2.6, we have in this case that

$$
\varphi(\omega) H(\omega)=\varphi(\omega)\left\{\int e^{-\omega t} d \mu(t)-\sum_{n=0}^{N} a_{n} \omega^{n}\right\}
$$

and

$$
\varphi(\omega) H_{1}(\omega)=\varphi(\omega)\left\{\int e^{-\omega t} d \mu_{1}(t)-\sum_{k=0}^{K} b_{k} \omega^{k}\right\}
$$

are analytic in the region enclosed by $C$. It might be possible for $\varphi(\omega)$ to have a pole at a zero of $H(\omega)$, except that $\varphi(\omega) H_{1}(\omega)$ is analytic at any such point and 
(excluding the origin) we know that $H_{1}(\omega)$ is not zero there. Hence the only possible pole of $\varphi(\omega)$ is at the origin, and the order of the pole is $\min \left(m, m_{1}\right)$.

By the definition of $f(z)$ in terms of $\varphi$ above, this makes $f(z)$ a polynomial of degree at most $\min \left(m-1, m_{1}-1\right)$.

This completes the proof of the theorem.

EXAMPLES. (I). Suppose $f(z)$ is entire of exponential type and $f(z+\tau)=$ $f(z)$ and $f\left(z+\tau_{1}\right)=f(z)$ where $\tau / \tau_{1}$ is irrational. If $d \mu$ and $d \mu_{1}$ are point mass measures at $-\tau$ and $-\tau_{1}$ respectively, the above periodicity equations become the convolution equations.

The functions

$$
\int e^{-z t} d \mu(t)-1=e^{\tau z}-1 \text { and } \int e^{-z t} d \mu_{1}(t)=e^{\tau_{1} z}-1
$$

each have a simple zero at the origin. This zero at the origin is the only common zero of the two functions, hence $f(z)$ is a polynomial of degree zero, i.e., $f(z)$ is a constant.

(II). Let $f(z)$ be entire of exponential type $a$ such that $f(x)=O\left(|x|^{J}\right)$ for some $J \geqslant 0$ as $|x| \rightarrow \infty$. Suppose further that $f(z)$ is periodic with period $\tau$ where $0<\tau<2 \pi a^{-1}$, i.e., $f(z)=f(z+\tau)$.

Let $d \mu=K a^{\prime}(t) d t$, where $K a(t)$ is as in (28), and let $d \mu_{1}$ be point mass at $-\tau$. From (28), we know that $f^{\prime}(x)=\int f(x-t) K a^{\prime}(t) d t$. We also found in that example that $\left(K a^{\prime}\right)^{\wedge}(t)=i t$ for $|t| \leqslant a$.

Thus the set of common zeros of $\left(K a^{\prime}\right)^{\wedge}(t)-i t$ and $\hat{\mu}_{1}(t)-1$ for $|t| \leqslant a$ is the set of zeros of $e^{i \tau t}-1$ for $|t| \leqslant a$. But $e^{i \tau t}=1 \Longleftrightarrow \tau t=2 k \pi, k=0, \pm 1$, $\ldots$, and by the choice of $\tau$ this only occurs at $t=0$ if $|t| \leqslant a$. Further, this root is simple. Thus $f$ is constant.

We see as an example that any entire function of exponential type 1 which is periodic with period $\tau<2 \pi$ is a constant.

\section{BIBLIOGRAPHY}

1. R. P. Boas, Jr., Entire functions, Academic Press, New York, 1954. MR 16, 914.

2. - Functions of exponential type. III, Duke Math. J. 11 (1944), 507-511. MR 6, 60 .

3. R. P. Boas, Jr. and H. Pollard, Continuous analogues of series, Amer. Math. Monthly 80 (1973), 18-25. MR 47 \#3903.

4. Louis de Branges, Hilbert spaces of entire functions, Prentice-Hall, Englewood Cliffs, N. J., 1968. MR 37 \#4590.

5. A. Friedman, Generalized functions and partial differential equations, Prentice-Hall, Englewood Cliffs, N. J., 1963. MR 29 \#2672.

6. A. O. Gel'fond, Linear differential equations of infinite order with constant coefficients and asymptotic periods of entire functions, Trudy Mat. Inst. Steklov. 38 (1951), 4267; English transl., Amer. Math. Soc. Transl. (1) 2 (1962), 337-366. MR 13, 929.

7. André Giroux, Une remarque sur l'interpolation des fonctions entières, Private communication, 1973.

8. I. Halprin and H. R. Pitt, Integral inequalities connected with differential operators, Duke Math. J. 4 (1938), 613-625. 
9. G. H. Hardy, Divergent series, Oxford Univ. Press, London, 1956.

10. David Jagerman, Information theory and approximation of bandlimited functions, Bell System Tech. J. 49 (1970), 1911-1941.

11. A. Kolmogorov, On inequalities between the upper bounds of successive derivatives of an arbitrary function on an infinite interval, UCen. Zap. Moskov. Gos. Univ. Matematika 30 (1939), 3-16; English transl., Amer. Matl. Soc. Transl. (1) 2 (1962), 233-243. MR 1, 298,$400 ; 11,86$.

12. Henry P. Kramer, The digital form of operators on band-limited functions, $\mathrm{J}$. Math. Anal. Appl. 44 (1973), 275-287. MR 48 \#8085.

13. E. Landau, Über einen Satz von Herrn Escalangon, Math. Ann. 102 (1929), 177188.

14. A. J. Macintyre, Laplace's transformation and integral functions, Proc. London Math. Soc. (2) 45 (1938), 1-20.

15. Thomas J. Osler, $A$ further extension of the Leibniz rule to fractional derivatives and its relation to Parseval's formula, SIAM J. Math. Anal. 3 (1972), 1-16. MR 48 \#2323a.

16. G. Pólya, Sur certaines transformations fonctionnelles lineaires des fonctions analytiques, Math. Z. 29 (1929), 549-640.

17. H. R. Pitt, On a class of integro-differential equations, Proc. Cambridge Philos. Soc. 40 (1944), 199-211. MR 6, 273.

18. Walter Rudin, Functional analysis, McGraw-Hill, New York, 1973.

19. I. J. Schoenberg, The elementary cases of Landau's problem of inequalities between derivatives, Amer. Math. Monthly 80 (1973), 121-158. MR 47 \#3619.

20. P. C. Sikkema, Differential operators and differential equations of infinite order with constant coefficients. Researches in connection with integral functions of finite order, Noordhoff, Groningen, 1953. MR 15, 623.

21. J. Tagamlitzki, Functionen, die auf der reellen Achse gewissen Ungleichungen genügen, Ann. Univ. Sofia Fac. Phys-Math. Livre 1, 42 (1946), 239-256. MR 9, 14.

22. J. M. Whittaker, Interpolatory function theory, Cambridge Univ. Press, London, 1935.

DEPARTMENT OF MATHEMATICS, SYRACUSE UNIVERSITY, SYRACUSE, NEW YORK 13210

Current address: Department of Mathematics, University of Santa Clara, Santa Clara, California 95053 